\title{
About Unitary Quantum Field Theory
}

\author{
L.G. Sapogin \\ Department of Physics, Technical University (MADI) \\ Leningradsky pr. 64, A-319, 125829, Moscow, Russia \\ Tel: 7-499-155-0492Ｅ-mail: sapogin@cnf.madi.ru
}

\begin{abstract}
The paper proposes a model of a unitary quantum field theory where the particle is represented as a wave packet. The frequency dispersion equation is chosen so that the packet periodically appears and disappears without changing its form. The envelope of the process is identified with a conventional wave function. Equation of such a field is nonlinear and relativistically invariant. With proper adjustments, they are reduced to Dirac, Schrödinger and Hamilton-Jacobi equations. A number of new experimental effects are predicted both for high and low energies.
\end{abstract}

Keywords: Wave packet, Dispersion, Unitary quantum theory, Corpuscular-wave dualism, Elementary particle, Unified field, Vacuum fluctuations.

\section{Introduction}

The most direct way of eliminating the existing theoretical difficulties in the relativistic interpretation of quantum-mechanical systems lies in the construction of a theory dealing only with a unified field, where are to be observed the quantities and the values that characterize that field at different points in time and space.

There is an impression that during the time since quantum theory was created, no substantial progress has been made in respect to our understanding of that theory. This impression is reinforced by the fact that neither field quantum theory nor the still imperfect theories of elementary particles have made any serious strides in the posing or solution of the following traditional questions:

- What are the reasons for the probabilistic interpretation of the wave function, and how can this interpretation be obtained from the mathematical formalism of the theory?

- What is really happening to a particle, when we "observe" it during interference experiments (for interference cannot be explained without invoking the particle "splitting-up" concept)?

- What is this statement in standard quantum mechanics really saying?: "a micro particle described by a point is the source of a field, but cannot be reduced to the field itself". Is it divisible or not? What does it really represent? Why is all of physics based on two key notions: point-particle as the field source and the field itself? Can only one field aspect remain, and still be considered as a physical entity that is as yet un-analyzable?

There are as yet no answers to these basic questions. "Exorcism" of the complementarity principle is irrelevant because that philosophy was invented ad hoc.

Many researchers think that the future of theoretical physics should be based upon a certain single field theory a unitary approach. In such a theory, particles are represented in the form of field wave clusters or packets. Mass would be purely a field notion, but the movement equations and all 'physical' interactions should follow directly from the field equations.

This is a very simple and heretofore unstudied possibility of formulating the unitary quantum theory for a single particle. Here we will deal only with the very general properties inherent in all particles and not touch upon the problems connected with such properties as charge, spin, strangeness, and charm.

After quantum mechanics appeared and was fully developed, a curious situation occurred: half of the founders of the theory clearly spoke out against it! Quoted below are a few of their remarks:

"The existing quantum picture of material reality is today feebler and more doubtful than it has ever been. We know many interesting details and learn new ones every day. But we are still unable to select from the basic ideas one that could be regarded as certain and used as the foundation for a stable construction. The popular opinion among the scientists proceeds from the fact that the objective picture of reality is impossible in its primary sense (i.e. in terms of images and movements- remark of author). Only very big optimists, among whom 
I count myself, take it is as philosophic exaltation, as a desperate step in the face of a large crisis. A solution of this crisis will ultimately lead to something better than the existing disorderly set of formulas forming the subject of quantum physics...If we are going to keep the damned quantum jumps I regret that I have dealt with quantum theory at all..." - Erwin Schrödinger.

"The relativistic quantum theory as the foundation of modern science is fit for nothing." - P. A. M. Dirac

"Quantum physics urgently needs new images and ideas, which can appear only in case of a thorough review of its underlying principles." - Louis de Broglie.

Albert Einstein, also, had the following to say:

"Great initial success of the quantum theory could not make me believe in a dice game being the basis of it...I do not believe this principal conception being an appropriate foundation for physics as a whole... Physicists think me an old fool, but I am convinced that the future development of physics will go in another direction than heretofore...I reject the main idea of modern statistical quantum theory... I'm quite sure that the existing statistical character of modern quantum theory should be ascribed to the fact that that theory operates with incomplete descriptions of physical systems only... ”- A. Einstein.

Although today the quantum theory is believed to be essentially correct in describing the phenomena of the micro-world, there is nevertheless experimental evidence - of cold nuclear fusion and mass nuclear transmutations, of anomalous energy sources and perhaps even perpetual mobile - which contradicts quantum theory.

When the real phenomenon of corpuscular-wave dualism was discovered, the first idea that occurred to Schrödinger was to present the particle as a packet of de Broglie waves. Later, British mathematician (C. Darwin, 1927) proved this idea to be wrong; as such wave packets would dissipate due to dispersion. Nevertheless, de Broglie studied a similar idea in a non-linear version, called the "double solution" (pilot wave) theory, until the end of his life.

The trouble with all previous attempts to present a particle as a field wave packet was that such a packet, according to proposed approaches, consisted of de Broglie waves. In our UQT approach, the packet consists of partial waves and the de Broglie wave appears as a side product during the movement and evolution of that partial wave packet.

Since we intend to describe physical reality by a continuous field, neither the notion of particles as invariable material points nor the notion of movement can have a fundamental meaning. Only a limited zone of space wherein the quantum field strength or energy density is especially large will be considered as a particle.

Let us conduct the following thought experiment: at the origin of a fixed coordinate system located in an empty space free of other fields, there is a hypothetical immovable observer, past whom a particle moves along the $x$ axis at a velocity of $v<<c$. Let us assume that the particle is represented by a wave packet creating a certain hitherto unknown field, and that the observer with the help of a hypothetical microprobe is measuring certain characteristics of the particle's field at different moments in time. This measuring is done on the assumption that the size of the hypothetical energy measuring device is many times less than the size of the particle and that it does not disrupt or influence the field created by this particle.

It is obvious that such an experiment is imaginary and cannot in principle be performed, but it doesn't prevent our imaginary device from being ideologically the simplest possible. In other words, we are interested in how the particle behaves and how it is structured when "no one is looking at it." Let the result of measurements at a certain point be function $f(t)$, describing the structure of the wave packet, the size of which is very small compared to the de Broglie wave. Knowing the particle's velocity $v$ and the structural function $f(t)$, the immovable observer can calculate the "apparent size" of the particle.

Let us assume that inside the corresponding wave packet the linearity of laws is not broken, and that the function $f(t)$ satisfies the Dirichlet conditions and can be split into harmonic components which we will call 'partial waves'. In using the complex form of development, we then obtain:

$$
f(t)=\sum_{s=-\infty}^{\infty} c_{s} \exp \left(i \omega_{s} t\right)
$$

where coefficients $c_{z}$ are the amplitudes of the partial harmonics (with the mean value of $C_{Q}=0$ ), and $\omega_{z}$ are the corresponding frequencies. To find the dispersion equation for partial waves, let us use the Rayleigh ratio for the group velocity $v$ of the wave packet: 


$$
v=v_{p}+k \frac{d v_{p}}{d h}
$$

Regarding the wave number $k$ of the partial wave as a function of the phase velocity $v_{p}$, let us integrate (1.2) with $v=$ const, since by the law of inertia the centre of the packet is moving at a constant speed. We will have:

$$
\kappa=\frac{\varepsilon}{\left|v_{p}-v\right|}
$$

where $C$ is the constant of integration. Integration was made on the assumption that velocity $v$ is constant and does not depend on the frequency of the partial waves, which follows from the experimentally derived law of inertia. If we assume that the particle is a wave packet, then its group velocity will be equal to the classical velocity of the particle. Since the particle is moving at a constant speed (inertial) in the absence of external fields, the group velocity of the packet is a constant value independent of the phase velocities of the harmonic components. The unsatisfying form of the dispersion equation (1.3) masks the linear dispersion law, which can

be derived from (1.3), by substitution of, $v_{\mathrm{p}}=\frac{w_{F}}{\vec{k}_{\mathrm{g}}}$ whereby:

$$
\omega_{8}=v k_{2} \pm C
$$

where plus sign corresponds to $v_{p} \geqslant v$ and minus sign corresponds to $v_{p}<v$. We will now define the

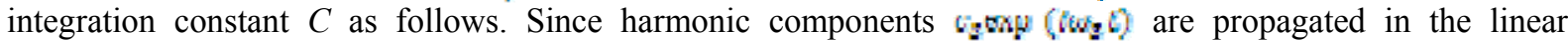
medium independently of each other, the behaviour of the wave packet can be presented as a superposition of the harmonic components:

$$
c_{s} \exp \left(i\left(\omega_{s} t-k_{s} x\right)+i \phi\right)
$$

Since the wave phase is now defined up to the additive constant, an additional constant $\phi$ for all partial waves was then introduced. Essentially, this is possible by simple translation of the origin of the coordinates, so the value $\phi$ can actually be excluded from further consideration. Then, the moving wave packet can be represented as follows:

$$
\Phi(x, t)=2 \Re e \sum_{1}^{\infty} c_{s} \exp \left(i\left(\omega_{s} t-k_{s} x\right)\right)
$$

Regarding the wave number $k(\omega)$ as a frequency function and substituting (1.4) into (1.6), we obtain:

$$
\Phi(x, t)=2 \Re e\left(\exp \left(-i\left(\frac{C}{v} x\right)\right) \sum_{1}^{\infty} c_{s} \exp \left(i \omega_{s}\left(t-\frac{x}{v}\right)\right)\right)
$$

or

$$
\Phi(x, t)=\cos \left(\frac{C}{v} x\right) f\left(t-\frac{x}{v}\right)+\sin \left(\frac{C}{v} x\right) f^{*}\left(t-\frac{x}{v}\right),
$$

where function $f^{*}\left(t-\frac{k}{v}\right)$ describes some additional partial waves with the same frequencies $\omega_{z}$. Analyzing expression (1.7), we can see that the wave packet $\phi(x, t)$ in its movement in a "medium" with linear dispersion described by equation (1.4) will disappear and reappear with period $\frac{\frac{2 \pi v}{G}}{\mathrm{~g}}$ in $x$ and can be regarded as if inscribed 
in the flat envelope modulating with that period. The state of the wave packet (and of its corresponding particle) in the region where it disappears or its amplitude becomes very small may be thought of as a "phantom state". Let us find integration constant $C$. For this, we will require that the wavelength of the monochromatic envelope be equal to the de Broglie wavelength:

$$
\lambda_{B}=\frac{2 \pi}{k_{B}}=\frac{2 \pi v}{C}
$$

Then, $C=v k_{B}$, and expression (1.7) will become as follows:

$$
\Phi(x, t)=\cos \left(k_{B} x\right) f\left(t-\frac{x}{v}\right)+\sin \left(k_{B} x\right) f^{*}\left(t-\frac{x}{v}\right) \cdot
$$

The disappearance and reappearance of the particle occurs periodically without change of its apparent dimensions (width and form). It is clear that the dimensions of each packet can be many times less than the de Broglie wavelength. An approximate picture of the behaviour of such a packet in space and time is presented in Fig. 1 below, and the results of the mathematical modelling of the scalar Gauss wave packet behaviour in a medium with linear dispersion are presented in Fig.2. The both figures show how such a packet disappears and reappears, changing its sign.

Any dispersion without dissipation leaves the packet's energetic spectrum unchanged. When the wave packet moves, only the phase relations between the harmonic components are changed, because dissipation is absent. This concept is based on two postulates:

(1) a particle represents a wave packet with linear field laws. The linear dispersion law follows from the law of inertia, and the particle is regarded as a moving wave packet inscribed in a flat envelope;

(2) the envelope wavelength is equal to the de Broglie wavelength. Nevertheless, any packets of de Broglie waves that are localized enough will be spread over the whole volume, as dispersion of the de Broglie wave

$\omega_{\varepsilon}=\frac{\sin \frac{2}{25}}{2 m}$ differs from linear dispersion. This does not contradict the suggested concept, as the envelope

doesn't exist as a real wave and is not included in the set of waves described by eq. (1.5).

Please note that the process of periodicity in the appearance and disappearance of the wave-packet/particle is possible only for very small objects, and that the quantum teleportation of macro-objects being widely discussed today is hardly possible by the principles under discussion here.

However, the theoretical possibility of the wave packet spreading in the transverse direction due to diffraction is still a concern. It is in principle that the packet can disperse and not exist as a localized formation. To show that this won't happen, let us put the equation of dispersion into another form. Viz., according to P. Ehrenfest, the theoretical envelope velocity of the wave packet equals the classical particle velocity:

$$
v=\frac{d \omega}{d k}=\frac{P}{m}
$$

On the other hand

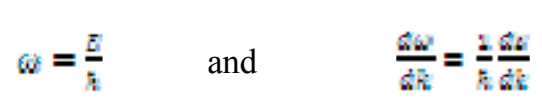

According to classical mechanics, the energy of a free particle is:

$$
E^{\prime}=\frac{F^{2}}{\Delta m} \quad \text { or } \quad \frac{d v}{d k}=\frac{E}{2 m} \frac{d E}{d k}
$$

Comparing (1.10) and (1.11) we obtain: 


$$
\frac{P}{\hbar m} \frac{d P}{d k}=\frac{P}{m},
$$

and by integrating that differential equation we get

$$
P=\hbar k+C .
$$

Now, the phase velocity of the waves,

$$
v_{p}=\frac{\hbar \omega_{s}}{\hbar k_{s}+C},
$$

does not remain a constant value but depends on constant of integration $C$.

By using another method to determine the velocity phase, the constant of integration may be added to the expression of energy (but this isn't a matter of principle). The choice of the constant of integration $C$ does not influence the results to be obtained in terms of quantum mechanics, and so for simplicity we assume that $C=0$.

The present conclusion represents a known fact about motion equation invariance as regards gradient calibrating transformation. The same relations for the phase velocity of quasi-particles also hold in solid-state physics, for quasi-particle momentum can be written as a constant divisible by the reciprocal lattice constant.

Let us return to (1.3). The choice of constant $C$ determines the type of dispersion. In the general case, that relation describes the wave set with different $k$ and $\lambda$. As we saw previously (and as is true in all inertial coordinate systems), with a certain type of dispersion the envelope of the de Broglie wave process is in a 'space-hold' condition. Putting $v_{p}=0$ in eq. (1.3), we obtain

$$
C=k v=\frac{m v^{2}}{\hbar} .
$$

Substituting the value for $C$ into this same expression (1.3) and taking into account that $k=\frac{w}{v}$, we will obtain the expression for subwave phase velocity:

$$
v_{p}=\frac{\hbar \omega_{s}}{ \pm m v+\frac{\hbar \omega_{s}}{v}}
$$

We should note that according to some works in quantum field theory, divergences are in principle eliminated by choice of $C$.

If the theory of wave transmission is linear, then the wave packet will diverge at the angle $\phi=\frac{\lambda}{b} \quad$ (Fig.3a).

Within the non-linear interpretation, one can see that self-focusing is able to compensate transverse diffraction (Fig.3b). For that to occur, the following relationship is necessary:

$$
v_{p}=\frac{c}{n}=\frac{c}{n_{0}+n_{2} E^{2}},
$$

where $c$ is light velocity. Then, the peripheral phase fronts bend toward the packet's axis, thus compensating transverse diffraction (as in Fig.3b above). As the wave packet's mass is proportional to the square of its amplitude, relation (1.12) can be rewritten in the following form:

$$
v_{p}=\frac{\hbar \omega_{s}}{ \pm m v+\frac{\hbar \omega_{s}}{v}}=\frac{c}{\frac{c}{v} \pm \frac{m v c}{\hbar \omega_{s}}}=\frac{c}{n_{0}+n_{2} E^{2}}
$$

provided $n_{0}=\frac{c}{v}, \quad n_{2}= \pm \frac{v c}{\hbar \omega_{s}}$, and $m \approx E^{2}$.

As yet we've said nothing about the nature of either the 'medium' or the waves propagating in it. In spite of various modern versions of quantum field theory, and the further development of UQT theory is impossible to answer at present the very simple question "what is space-time?" Is it simply the "stage" where performers in the 
form of a multi-component field are continually appearing and disappearing? Or does the field represent dynamic distortions of the stage itself, so that it's impossible to separate the performers from that stage?

\section{Equations of Unitary Quantum Theory}

The wave function of a single particle (1.9) was derived on an assumption of non-relativistic velocities, i.e., for $\mathrm{v}<<\mathrm{c}$. To obtain its relativistic generalization it is first necessary to make the wave function as a relativistically invariant phase, (L.Sapogin, 1979, 1980) i.e.,

$$
\Phi=\exp [-i(E t-\mathbf{P x})] \mathbf{f}(\mathbf{x}-\mathbf{v} t),
$$

where

$$
E=\frac{m}{\gamma} ; \mathbf{P}=\frac{m \mathbf{v}}{\gamma} ; \gamma=\sqrt{1-v^{2}}
$$

And $\mathbf{f}(\mathbf{x}-\mathbf{v} t)$ is some structural function (in this paragraph, we use a unit system in which $c=\hbar=1$ ). It can be required that structural function $\mathbf{f}(\mathbf{x}-\mathbf{v} t)$ be scalar and satisfy the Klein-Gordon equation. Then, we will get the following equation for $\mathbf{f}$ :

$$
\left(v_{i} v_{k}-\delta_{i k}\right) \frac{\partial^{2} \mathbf{f}}{\partial \xi_{i} \partial \xi_{k}}=0
$$

Here, $\xi_{i}=x_{i}-v_{i} t ; i, k=1,2,3$, and summarization is obtained by repeated indices as usual. A two-component solution of the Klein-Gordon equation would then appear as follows:

$$
\Phi=\exp (-i(E t-\mathbf{P x}))\left(\begin{array}{l}
\frac{\gamma-1}{2 \gamma} \mathbf{f}-\frac{i}{2 m} \mathbf{v} \frac{\partial \mathbf{f}}{\partial \xi} \\
\frac{\gamma+1}{2 \gamma} \mathbf{f}+\frac{i}{2 m} \mathbf{v} \frac{\partial \mathbf{f}}{\partial \xi}
\end{array}\right)
$$

By substituting (2.1) into the Schrödinger equation we may obtain the Laplace equation for structural function as:

$$
\nabla_{\xi}^{2} \mathbf{f}=0,
$$

and its solution will enable us to regard the particle as a spherical wave packet "cut into parts" by spherical harmonics.

But such an approach can only serve as a certain illustration, a first approximation based on the assumption of field law linearity. Function $\mathbf{f}$ described by the Laplace equation will tend to infinity at zero, which is completely unsatisfactory from the physical point of view. Let us do otherwise, and consider just the simplest equations of first and second order, which are satisfied by a one-component relativistic wave function having an arbitrary structural function. These equations have a clearly relativistic form:

$$
\begin{gathered}
\left(u_{\mu} \frac{\partial}{\partial x_{\mu}}+i m\right) \Phi=0 \\
\left(u_{\mu} u_{v} \frac{\partial^{2}}{\partial x_{\mu} \partial x_{v}}+m^{2}\right) \Phi=0
\end{gathered}
$$

where: $x_{\mu}=(\mathbf{x}, i t) ; u_{\mu}=\left(\frac{\mathbf{v}}{\gamma}, \frac{i}{\gamma}\right)$ is the particle's four-velocity; and $\mu, v=1,2,3,4$. It is natural to consider that a particle with an arbitrary spin and mass $m$ can be described by a relativistic equation

$$
\left(\Lambda_{\mu} \frac{\partial}{\partial x_{\mu}}+m\right) \Phi=0
$$

where $\Phi$ is an $n$-component column and $\wedge_{\mu e}$ represents four ( $\left.\mathrm{n} \times 4\right)$ - matrices (n-rows, 4-column) describing the spin properties of the particle. These matrices are functions of the particle velocity and satisfy relations that are defined by the spin value. 
Let us now express particle energy (mass) by means of a field. For Dirac-type equations, neither the character of charge with an integer spin nor charge energy with half-integer spin are defined. In relativistic electrodynamics, according to the Laue theorem, the tensor components of the energy-impulse of the electromagnetic field that is generated by the charge do not form four-vectors, so there is only one method of expressing the particle energy:

$$
E=m=\int_{V} \Phi^{+} \Phi d^{3} x
$$

Usually in such cases it is required that the integral (2.6) contain the Green function. However, if we strictly follow the principles of the unitary theory, we should define the particle energy within non-relativistic limits as in expression (2.6).

Let us substitute the invariant relativistic expression $\langle\Phi \mid \Phi\rangle$ for $\int_{V} \Phi^{+} \Phi d^{3} x$, which, for example, equals (O.Costa de Beauregard, 1957) for a spin field with a rest mass differing from zero (there are also formulas for the scalar and vector fields):

$$
\langle\Phi \mid \Phi\rangle=\int\left\{\Phi^{*} i \gamma_{4} \frac{\partial}{\partial t} \hat{\varepsilon} \Phi-\frac{\partial}{\partial t} \Phi^{*} i \gamma_{4} \hat{\varepsilon} \Phi\right\} d V
$$

where $\gamma_{4}$ is a Dirac matrix, $\hat{\varepsilon}=+1$ for a particle, and $\hat{\varepsilon}=-1$ for an antiparticle. Then, eq. (2.5) will look as follows:

$$
\left\{\Lambda_{\mu} \frac{\partial}{\partial x_{\mu}}+\langle\Phi \mid \Phi\rangle\right\} \Phi=0
$$

This nonlinear integro-differential equation is, in our view, fundamental, and must describe all the properties and interactions of particles (L.Sapogin, 1979, 1980, 2005, 2008, 2010), (V.Boichenko, 1984). The mass spectrum from such equations may be derived after solving stability problems of the Sturm-Liuville type, which will in turn give the particle lifetime. In the theory under consideration, the birth and decay of all particles, and all of their interactions and transformations, are consequences of wave packet splitting and mutual diffraction phenomena due to nonlinearity. The construction of solutions to that problem will plainly require some new mathematical methods.

Point-like particles may be required to simplify the solution of the preceding eq. (2.8), whereby it reduced to the main equation of nonlinear (W.Heisenberg,1966) theory written not in operator form but in $c$-numbers. For this it is necessary in eq. (2.5) to substitute $m=\Phi^{+} \Phi$. Then we obtain the following equation

$$
\left(\Lambda_{\mu} \frac{\partial}{\partial x_{\mu}}+\Phi^{+} \Phi\right) \Phi=0,
$$

and there was derived an approximate particle mass spectrum with help of this equation.

Let us pass from equation (2.5) to the equation of particle motion in an external electromagnetic field $A_{\mu}$. We will therefore make a standard substitution $\frac{\partial}{\partial x_{\mu}} \rightarrow \frac{\partial}{\partial x_{\mu}}-i e A_{\mu}$, and eq. (2.5) is transformed as follows:

$$
\left(\frac{\partial}{\partial t}+\mathbf{v} \frac{\partial}{\partial \mathbf{x}}-i L\right) \Phi=0
$$

where $L$ is a relativistic Lagrangian,

$$
L=m \gamma+e \gamma U_{\mu} A_{\mu}
$$

If a particle is located in an external electromagnetic field, for example, with vector potential $\boldsymbol{A}$ and scalar potential $\varphi$, then the linear dispersion law is not changed. $L$ and $\mathbf{v}$ will then be certain functions of coordinates and the solution of eq. (2.10) in a general form has the following form: 


$$
\Phi=\exp \left(-i \int L d t\right) \mathbf{f}\left(\mathbf{x}-\int \mathbf{v} d t\right)
$$

It is easy to make a standard transition from the relativistic case to the non-relativistic case by using the well-known transformation $\Phi=\Phi e^{-i m t}$. Substitution of function (2.11) into the equation (2.10) shows that the equation is satisfied provided $L$ is a non-relativistic Lagrangian. Let us now look at the role of the wave function phase, which is the classic action $S$ and will enable us to establish a connection between the proposed theory and classical mechanics. Actually, the wave function may be represented in the form below (following Hamilton's principle in classic mechanics):

$$
\Phi=\exp (i \mathrm{~S}) \mathbf{f}\left(\mathbf{x}-\int \mathbf{v} d t\right)
$$

If we substitute this expression into eq. (2.10), we then obtain an equation for $S$ :

$$
\frac{\partial S}{d t}+\mathbf{v} \nabla S-L=0
$$

In keeping with the requirements of the Hamilton-Jacobi theory, it is necessary to assume that $\mathbf{P}=\nabla S$; then eq. (2.12) will be transformed to the Hamilton-Jacobi equation:

$$
\frac{\partial S}{\partial t}+H=0,
$$

where $H=\mathbf{P v}-L$ is the particle's Hamiltonian.

The function $S$ can thereby be found, dependent on the particle's coordinates, the physical parameters of the Hamiltonian, and on $q$ non-additive integration constants; and then perhaps the problems of motion and dynamics can be solved. The imposed requirement $\mathbf{P}=\nabla S$ implies a transposition to classic mechanics using an optic analogy approximation, whereby the concept of particle trajectory as a beam can be introduced. Such a trajectory will be orthogonal to any given surface of a permanent operation or phase.

On the other hand, a quantum object becomes a classical construct after superposition of a large number of wave packets. The case where all wave packets composing an object spread and reintegrate simultaneously despite different velocities and phases is physically impossible. That is why such a combination when averaged out will appear, in general, like a stable and unchanging object moving under the laws of classical mechanics, whereas every elementary object obeys the quantum laws.

Note that a transfer from the unitary quantum theory to classical mechanics is mathematically strict. In the usual quantum theory, the transfer happens with an imposed condition $A-0$. Mathematically, it is completely unsatisfactory, since $\hbar$ is some physical constant (equal to 1 if given a corresponding units system). We do not remember a single case in mathematics when a similar condition would be imposed in a proof, such as $\pi \rightarrow 1$. Let us consider briefly the hydrogen atom problem. The solution of classical problem of particle movement in the central field allows to present the wave function (2.1) as follows:

$$
\Phi=e^{-i E t} e^{i \int_{0}^{r} p_{r} d r} e^{i \int_{0}^{\phi} p_{\phi} d \phi} f\left(\mathrm{r}-\int_{0}^{t} v_{r} d t ; \phi-\int_{0}^{t} \phi d t\right)
$$

Here, $r_{0}$ and $\phi_{0}$ are particle coordinate values (radius and angle correspondingly) at time $t=0$. Stationary orbits appear when the envelope is a standing wave provided:

$$
E T=2 \pi n_{1} h ; \oint p_{r} d r=2 \pi n_{2} h ; \oint p_{\phi} d \phi=2 \pi n_{3} h,
$$

where $n_{1}, n_{2}, n_{3}$ are integers. These requirements correspond to the terms of Bohr-Sommerfeld quantification.

The process envelope can be identified with the de Broglie wave and in essence the Schrödinger equation describes the envelope of the wave packet's maxima in motion. In conclusion of this section, let us find matrices $\Lambda_{\mu}$. Let us assume that matrices $\Lambda_{\mu}$ are linear relative to velocity: 


$$
\Lambda_{\mu}=\Lambda_{\mu 0}+\Lambda_{\mu v} u_{v}
$$

where $\Lambda_{\mu 0} \times \Lambda_{\mu v}$ are numerical matrices. Let us apply equation (2.5) on the left with operator $\Lambda_{\sigma} \frac{\partial}{\partial x_{\sigma}}-m$, obtaining:

$$
\left\{\frac{1}{2}\left(\Lambda_{\mu} \Lambda_{\sigma}+\Lambda_{\sigma} \Lambda_{\mu}\right) \frac{\partial^{2}}{\partial x_{\mu} \partial x_{\sigma}}-m^{2}\right\} \Phi=0
$$

If we require that each component of system (2.14) satisfies the second order equation (2.4), and then

$$
\Lambda_{\mu} \Lambda_{\sigma}+\Lambda_{\sigma} \Lambda_{\mu}=-2 u_{\mu} u_{\sigma} I
$$

Relation (2.15) is satisfied identically if we take ten Hermitian matrices $32 \times 32$ as numerical matrices $\Lambda_{\mu \omega}$, satisfying the following commutation relations:

$$
\Lambda_{\mu \nu} \Lambda_{\sigma \tau}+\Lambda_{\sigma \tau} \Lambda_{\mu \nu}=2\left(\delta_{\mu \sigma} \delta_{v \tau}-\delta_{\mu \tau} \delta_{v \sigma}\right) I
$$

Here, indices $\xi_{i}, \sigma, \tau$ take values $0,1,2,3,4$. It is interesting to note that if the particle's 4-velocity is assumed to be zero $\left(w_{i k}=0\right)$, then system (2.5) will reduce to eight similar Dirac equations. However, this requirement is absolutely unsatisfactory both from the physical and the mathematical points of view. Four-velocity has 4 components, of which three are usual components of the particle velocity along three axes, and they really can tend to zero. But the same cannot be done with the fourth component.

Hence, this approach is formally incorrect and requires explanation. In our view, although the Dirac equation describes the hydrogen atom spectrum absolutely correctly, it is not properly a fundamental equation. It has two weak points:

1. The correct magnitude of the velocity operator's proper value is absent. It is known that in any problem of this type the proper value of the velocity operator is always equal to the velocity of light! In fact, Russian physicist and mathematician V. A. Fock regarded this as an essential defect of the Dirac theory;

2. The Klein paradox appears in the solution of the problem of barrier passage, when the number of the particles that pass is bigger than the number of incident particles.

The equations of the Unitary Quantum Theory we are proposing are more correct and fundamental. For this reason, a transition from correct fundamental equations to the incompletely accurate Dirac equation needs such a strange requirement as $w_{\mu k}=0$.

\section{Interpretation of Unitary Quantum Theory}

\section{Non-Relativistic Case}

The envelope of the wave function $\Phi(x, t)$ describes a wave packet's field transformation. within its motion. There are points at which the packet/particle disappears, $\Phi(x, t)=0$, yet particle energy remains in the form of harmonic components that produce field vacuum fluctuations at some point in space-time. Neither the value and moment of these fluctuations' appearance nor the background flux at that point depend on the apparent distance to such a vanished. particle. This precept does not violate the principles of relativity, however, in that the apparent background does not transfer any information.

Our real 'world' continuum consists of an enormous quantity of particles moving with different velocities. Partial waves of the postulated vanishing particles create real vacuum fluctuations that change in a very random way. Certain particles randomly appear in such a system, owing to the harmonic component energy of other vanished particles. The number of such "dependant particles" changes, though; they suddenly appear and vanish forever, as the probability of their reappearance is negligibly small, and so we do expect that all particles are indebted to each other for their existence. Yet, if some particles are disappearing within an object, other particles are arising at the same moment in that object due to the contribution of those vanishing particles' harmonic components - and vice versa. The simultaneous presence of all of the particles within one discrete macroscopic object is unreal. Some constituent particles vanish within the object while others appear. In general, a mass object is extant overall, but is not instantaneously substantive and merely a 'false' image. All Universe is 
mathematical focus. It is clear that the number of particles according to such a theory is inconstant and all their ongoing processes are random, and their probability analysis will remain always on the agenda of future research.

In reality, the hypothetical measurements considered before are impossible, because all measuring instruments are macroscopic. Since the sensor of any such device is an unstable-threshold macro-system, only macroscopic events will be detected, such as fog drops in a Wilson chamber, blackening of photo-emulsion film, photo-effects, and the formation of ions in a Geiger counter. Within macro-devices of any type, the sensor's atomic nuclei and electron shells are in close proximity, creating a stable system which is far from being able to take on all arbitrary energy configurations that might be imagined.

The nature of that stable condition allows for only a series of numerous but always-discrete states, and the transition from one state to another is a quantum jump. This is why absorption and radiation of energy in atomic systems takes place by quanta, and is a consequence of subatomic structure. In other words, quantization appears because of the arising of bound states, with 'substance' being the richest collection of an enormous number of bound states. However, it is known that free particles may vary their energy continuously.

However, this does not mean that while passing from one quantum-mechanical system to another, the quantum or particle remains as something invariable and indivisible. Particle energy can be split up and changed due to vacuum and external field fluctuations, but the measuring conditions of our devices are such that we are able to detect quite definite and discrete particles only.

The wave packet/particle exhibits periodicity following our UQT approach, and the mass of a moving particle such as a proton changes from its maximal value to zero and back again - running the series of intermediate values corresponding to the masses of mesons. For example, it might be said that the proton takes, during some intervals of time, the form of a $\pi$-meson. This phenomenon is confirmed by numerous experiments, which are explained in classical quantum theory in another way: The proton is permanently surrounded by a cloud of $\pi$-mesons, an explanation which is in essence equivalent to our model.

Thus the developing point of view results in the conclusion that relation $\bar{E}=\hbar \boldsymbol{L} \omega$ is fulfilled at the atomic level only. Thus the particles may exist (after fragmentation on the mirror) with similar frequency $w_{z}$, but with different wave amplitudes $f$, and so with different probabilities to be detected. One of the particles being split up at the mirror or grid may be detected in a few points at once. The other particle may disappear completely, making its contribution in vacuum fluctuations without any marks.

Following P. Dirac, the photon may interfere only on its own and so the translucent mirror splits it into two parts. According to standard quantum theory, the photon is not able to split with frequency conservation, so it is assumed that two separate photons may interfere under the condition that they belong to one mode, which occurs in the case of the translucent mirror. However, according to UQT, photons are constantly splitting at the translucent mirror with frequency conservation, but the probability to detect such splitting photons is reduced.

An uncertainty relation results from the fact that energy and impulse are not fixed values, but periodically change due to the appearance and disappearance of the particle. That question is examined in detail in sect. 7 . Due to the statistical measuring laws, it is impossible to measure energy and impulse by macro-devices exactly because of principal and not-foreseen vacuum fluctuations. On the other hand, for the hypothetical researcher the centre of the wave packet has exact coordinates, impulse, and energy at the given moment of time. However, neither we nor the hypothetical observer are able to predict exactly its value at the following moment. Moreover, we (macro-researchers) do not have even a method of accurate measuring, for the process of macro-devices measuring is statistical.

The presence of vacuum fluctuations makes microcosm laws for each researcher statistical in principle. The exact prediction of the events requires the knowledge of the vacuum fluctuation's exact value in any point and at any moment of time. This is impossible, for it requires the information about behaviour and structure of all various wave packets within the Universe and also the possibility to control their motion.

(W. Heisenberg, 1966) wrote : "If we would like to know the reason why $\alpha$-particles are emitting at an exact moment we must, apparently, know all microscopic states of the whole world we also belong to, and that is, obviously, impossible."

This is why the conclusion that Laplace determinism is lost within the modern and future physics of microcosm shall be considered ultimate. The same point of view about the reason of the arising of probability approach in quantum mechanics was expressed by (R. Feynman, 1965) : "There is almost no doubt that it (probability-LS) 
results from the necessity to intensify the effect of single atomic events up to the level detectable with the help of big systems."

It is good to remember the deep and remarkable words of J. Maxwell: "The calculation of probabilities is just the true logic of our world."

The most impressive demonstration of the random chaotic nature of all quantum processes can be seen at the start of a nuclear reactor. Chaos of micro-effects at a low level of average power results in enormously huge fluctuations of chain reactions, which exceed to a considerable extent the average level. Atomic chaos manifestations always exasperate the participants and sometimes create a threatening impression of the processes' uncontrollability with all following consequences. However, cadmium rod removal precipitates smoother fluctuations.

The envelope of partial waves appearing in the result of wave packet linear transformations and also in the result of it splitting and fragmentation satisfies the $\mathrm{C}$. Huygens principle. This explains the way it is possible to connect the formally moving particle and plane monochromatic de Broglie wave as it spreads in the line of motion and also all the wave properties of particles (such as interference and diffraction).

For example, let the wave packet run up to the system with two slots. Each of the wave packet harmonic components interferes at these slots. There would be an interference pattern of each harmonic component at the screen (since harmonic components amplitudes are extremely small, it may be not possible to see it). However, above this interference pattern the other interference patterns of an infinite large number of the other harmonic components are superimposed. The general composition results in the long run interference pattern of the de Broglie wave envelope.

For the total reversibility of quantum processes, it is necessary while exchanging $+t$ for $-t$ not only to reproduce the amplitude and form of the packet at $+t$, but also to restore the background fluctuation. The quantum mechanics equations permit formal exchange $+t$ for $-t$ under the condition of simultaneous exchange $\Phi$ for $\Phi^{*}$, i.e. formal reversibility (the amplitude and form of the packet reproduction). Actually, such reversibility does not exist in nature even for the hypothetical observer, as for reproduction of the former vacuum fluctuations the reversibility of all processes in the Universe is required, and that is impossible. However, one is able to think that in terms of Unitary Quantum Theory the reversibility has a statistic character (single processes may be reversible with define probability).

Introduced function $\Phi$ has a strictly monochromatic character, but does not exist as a real plane running wave. Although this function corresponds to the particle's energy, other notions may also agree with it: "Waves of probability", "informational field", and "waves of knowledge". As stipulated by (A. Alexandrov et al., 1966) a wave function has sense for a separate system, but we can pick it out only by the way of numerous similar experiments and after averaging, though the hypothetical researcher is able to measure this wave function for one particle. It is interesting that the envelope remains fixed within all inertial coordinates systems (only the wave length is changed).

Function $\Phi$ may also be connected with wave function $\Psi$ of quantum mechanics describing the plane wave moving in the space. However, the value $\Phi^{2}$ differs from $\Psi \Psi^{*}$ not only by presence of frequent oscillations. With $\Phi^{2}$ the particle's energy is connected, but with $\Psi \Psi^{*}$ only the probabilities connect.

In standard quantum theory all is not so easy. When comparing mathematical expressions for the density matrix in quantum mechanics and the correlation function of random classical wave field, then we find them quite similar, although they describe absolutely different physical objects. In the simplest cases the wave function relates to a single particle and has any sense in the presence of the particle only. Wave function has no sense in those areas where particle is absent. More formally, according to quantum theory, physical values can be obtained in the result of either one or other operators' acts on wave function. Then the average values may be computed by averaging with some weight. That is why notions of absolute phases and amplitudes have no physical sense and may be selected arbitrary for usability only. Large relative changes of the amplitude in far situated points do not result in physical values changes if the wave function gradient is being transformed slightly. So $\Psi^{2}$ have a probability distribution sense but not the sense of real wave motion density as it were in the case of classic fields.

In contrast to ordinary quantum theory the phase plays quite an essential role according to our approach. For example, if a particle reaches the potential barrier being in phase of completely vanishing $(\Phi(x, t)=0)$, then due to linear character and superposition at small $|\Phi|$ it penetrates the quite narrow barrier without any interactions (Fig.4). 
At the other hand, if the phase is so that value of $|\Phi(x, t)|$ is maximal, then due to non-linear character interactions would began and the particle might be reflected. That idea results in new effect: if there were a chain of periodical (with period $a$ ), narrow enough (in comparison with $\lambda_{B}$ ) potential barriers, bombarded with monochrome particles flux, then abnormal tunnelling is to be considered at $\lambda_{B}=2 a$, that does not exist in standard quantum theory.

Mathematically the process of the packet's appearing and vanishing without changing its character is possible as it is shown at Fig.1. It enables formally to understand the fundamental fact of two different amplitude interference rules: for bosons when amplitudes interfere with equal signs and for fermions - with different signs (Fig.4).

\section{Relativistic case}

Analyzing (2.1) one can see that wave packet $\Phi$ contains oscillations term with frequency $\omega_{z}=\frac{m_{s}^{2}}{k_{y}^{2}}$ that corresponds to Schrödinger vibration. The physical meaning of that very quick oscillating process is the follows: after "Creator" having stirred up "the medium" created wave packet the last began oscillating like membrane or string with frequency $\omega_{z}$. Within the motion there arising de Broglie vibrations with frequency $\omega_{z}=\frac{w_{w}^{2}}{R_{p}}$ due to dispersion. At small energies $\omega_{\xi} \omega_{F}$ and in the presence of quick own oscillations have no influence on experiment and all quantum phenomena result from de Broglie oscillations. The value of frequency $\omega_{\bar{\Xi}}$ tends to $\omega_{3}$ with growth of energy and resonance phenomenon appears that result in oscillating amplitude increase and in mass growth (Fig. 5). Thus the well-known graph of particle mass dependence on the velocity approaching to light's velocity constitutes actually a half of usual resonance curve for forced oscillation of harmonic oscillator if energy dissipation is absent. In the case when $v \rightarrow c$, frequency $\omega_{\varepsilon} \rightarrow \omega_{g}, \gamma \rightarrow 0$ beats appear with resonance frequency $\omega_{\alpha}=\omega_{3}-\omega_{z} \approx \frac{\omega_{0}^{2} c^{2}}{a}$, and particle will obtain absolutely new low-frequency envelop with wave length

$$
\Delta=\frac{h}{n \in C}
$$

This is a new wave. In ultra-relativistic limit case the value of $\mathrm{A}$ becomes much greater as typical dimension of quantum system it (new wave) interacts with. Now the length of new wave grows with energy contrary to de Broglie wave length slowly decreasing, and particle requires the form of quasi-stationary wave packet moving in accordance with classical laws. That explains the success of hydrodynamics fluid theory concerning with numerous particle birth when the packet having extremely big amplitude is able to split into series of packets with smaller amplitudes. But such splitting processes characterize not only high-energy particles. Something like this takes place at small energies also, but overwhelming majority of arising wave packets are under the barrier and so will not be detected. It would be perfect to examine by experiments at future accelerators the appearance of such new wave with the length growing together with energy.

But there is once more sufficiently regretting considerations. Due to our point of view relativistic invariance of equations should be apparently changed for something else. The fact is that classical relativistic relation between energy and impulse

$$
E^{2}=P^{2}+m^{2}
$$

does not working for extra short intervals of time and small particle's displacement (equal to parts of de Broglie wave length). This relation is the result of averaging. What happens with particle impulse and mass when the packet has been spread all over the Universe? Possibly they go to zero, but particle's energy as integral of all harmonic components squares sum remains constant (no wave dissipation) and the above-mentioned relation breaks. And probably the fundamental equation (3.2) should be written in any other form. But to be sure that equation should be solved first. 


\section{The Theory of Optimal Detector and Quantum's Measurements}

Any 'normal' measurement, in the long run, is based on the interchange of energy and is an irreversible process. That is why the particle interferes in the state of macro-device giving up (or acquiring in the case of devices with inversion) quantum of energy $\theta$. The best measuring instrument would be one wherein the discrete threshold energy $\theta$ which characterizes device instability was absolutely minimal. With a hypothetical measurement $\theta=$ 0 , such that the researcher does not influence the particle with his sensor, then such a device would have $100 \%$ effectiveness and could detect any vacuum fluctuations.

The measuring instrument should be so that eventually only its classical characteristics were used for its work; in other words, Planck's constant should not play any role in it after the initiation. Such a device is as much as possible (but not totally) free from statistical effects. Thus in measuring processes particle detectors are those reference frames in what respect according to the quantum theory the system's state is to be determined.

Let us consider the process of particle - macro-device interaction (L.Sapogin, 1982, 1982, 2003, 2005, 2008). Particle energy periodically changes with frequency $\omega_{B}$ and vacuum fluctuations (additionally changing the energy) are imposed at it in a random way. To detect the particle, the macro-device has to wait until particle total energy $|\Phi|^{2}$ and vacuum fluctuations $\varepsilon$ exceeds the operation threshold $\theta$ of the device:

$$
\varepsilon+|\Phi|^{2} \geq \theta
$$

The energy of vacuum fluctuation $\varepsilon$ depends on the total number of the particles in the Universe and is created thanks to the particles disappeared. As far as the contribution of each partial wave in every point is infinitesimal (its distribution law may be any) in accordance with Central Limit Theorem of Alexander Lyapunov the summary background to be formed by tremendous number of particles and their partial waves will have a normal distribution with maximal entropy. The probability $P$ of vacuum fluctuations with the energy more than $\varepsilon_{0}$ is equal to

$$
P=\frac{1}{\sqrt{(2 \pi)} \sigma} \int_{\varepsilon_{0}}^{+\infty} \exp \left(-\frac{\varepsilon^{2}}{2 \sigma^{2}}\right) d \varepsilon
$$

and the value $\sigma$ (dispersion), depending on the particles' number within the Universe is considered in our case as constant. The theory under consideration requires finiteness of $\sigma$, and then finiteness of the Universe. It is evident from the last formula that the probability of the particle's detecting depends on the sensitivity of the measuring instrument.

Without entering into detail of the interaction between quantum particles with macro instruments, the problem of particle recording or detection can be stated as follows: On a wave packet with value $|\Phi|$ a vacuum fluctuation with value $\varepsilon$ is additively imposed. For simplicity, let us regard the problem as single-dimensional and the eigenregion of the field as a segment of the numerical axis. Mark on that axis $x$ a certain threshold value (Fig. 6)

$$
\theta<a=|\Phi|
$$

and let the eigenregion of the acting field be $\theta<x<\infty$. The measuring macro instrument distinguishes two situations. If there is a particle, then the value of the field which acts on the instrument is $a+\varepsilon$; if there is no particle, the value is $\varepsilon$. The instrument responds (the particle is recorded) when the value of the acting field exceeds a certain threshold $\theta$, and then $\theta^{2}$ is the minimal quantum energy for the macro-instrument to respond (sensitivity).

Let us find the probability of error of the instrument. Let the distribution of vacuum fluctuations $W_{a}(x)$ be the distribution of the sum of the particle field and vacuum fluctuations $W_{0}(x)$. The conditional probability of failing to detect a particle when this goes through the macro instrument is (it is the case of $\theta=\theta_{1}$ in Fig.6)

$$
p_{a}(0)=p\{a+\varepsilon<\theta\}=\int_{-\infty}^{\theta} W_{a}(x) d x
$$

and the conditional probability of detecting a particle when it is not there is 


$$
p_{0}(a)=p\{\varepsilon>\theta\}=\int_{\theta}^{\infty} W_{0}(x) d x
$$

Let $p(a)$ and $p(0)$ be a priori the probabilities of particle flight or absence. Then the total probability of error is

$$
p_{\text {error }}=p(a) p_{a}(0)+p(0) p_{0}(a)=p(a) \int_{-\infty}^{\theta} W_{a}(x) d x+p(0) \int_{\theta}^{\infty} W_{0}(x) d x
$$

An instrument whose $F_{\text {errar }}$ is minimal can be viewed as optimal. When the threshold $\theta$ is lowered, the instrument sensitivity increases and thus the number of undetected particles is reducing, but the vacuum fluctuations increase the number of false recordings. When the threshold $\theta$ is increased, the number of false recordings decreases, but the number of undetected particles increases. It is intuitively clear that, at some value of the threshold $\theta$, the value must go down to minimum (Fig. 6). Let us find that

$$
\frac{d p_{\text {error }}}{d \theta}=p(a) W_{a}(\theta)-p(0) W(\theta)=0
$$

Assuming for simplicity that $p(a)=p(0), a=$ Const we have

$$
W_{a}(\theta)=W_{0}(\theta), \quad W_{a}(x)=W_{0}(x-a)
$$

and

Since $W_{0}(x)$ is an even function,

$$
W_{0}(\theta)=W_{0}(\theta-a)
$$

$$
W_{0}(\theta)=W_{0}(a-\theta)
$$

hence

$$
\theta=\frac{a}{2}=\frac{|\Phi|}{2} ; \quad \theta^{2}=\frac{1}{4}|\Phi|^{2} .
$$

Consequently, for the optimal quantum detector the threshold energy should be one-fourth of the particle energy. Usually this relation does not hold and inequality is true $\theta^{2} \leq q^{2} \Phi$ or the number of false recording is very high. In compliance with relation (4.3) the normalizing condition

$$
\int_{-\infty}^{+\infty} W_{0}(x) d x=1
$$

and by assuming that the flight of the particle or its absence are equiprobable events $P(Q)=P(0)=\frac{1}{2}$ expression (4.3) can be transformed:

$$
p_{\text {error }}=\frac{1}{2}\left(\int_{-\infty}^{\frac{a}{2}} W_{a}(x) d x+\int_{\frac{a}{2}}^{+\infty} W_{0}(x) d x\right)=\int_{\frac{a}{2}}^{\infty} W_{0}(x) d x=\frac{1}{2}-\int_{0}^{\frac{a}{2}} W_{0}(x) d x
$$

After introducing a new variable $y=x / \sigma$, where $\sigma$ is the r.m.s. of vacuum fluctuations, being normally distributed, we obtain

$$
\begin{aligned}
& p_{\text {error }}=\frac{1}{2}-\int_{0}^{\frac{a}{2 \sigma}} V_{0}(y) d y, \\
& V_{0}(y)=\frac{1}{\sqrt{2 \pi}} \exp \left[-\frac{y^{2}}{2}\right] .
\end{aligned}
$$

Thence, 


$$
p_{\text {error }}=\frac{1}{2}-\frac{1}{\sqrt{\pi}} \int_{0}^{\frac{a}{8 \sigma}} \exp \left(-z^{2} d z\right)=\frac{1}{2}\left(1-e r f \sqrt{\frac{a^{2}}{8 \sigma^{2}}}\right)
$$

Then the error of the detectors is small and expressed as a fraction of the form $P_{\operatorname{arr}}=10^{-\frac{p}{p}}$ where $P=0 \ldots 6$ for

most existing instruments. Denoting $\rho=\frac{a^{2}}{\alpha^{2}}$ we have the probability of detecting the particle, if it exists, in the

form

$$
P=-\log \frac{1}{2}\left(1-\operatorname{erf} \sqrt{\frac{\rho}{8}}\right)=-\log \frac{1}{2}\left(1-\operatorname{erf} \frac{\operatorname{Re} \Phi}{\sqrt{8 \sigma^{2}}}\right)
$$

This is the interpretation of a wave function in unitary quantum theory. The relation $P(\rho)$ does not make an impression until a plot of $P(\rho)$ is seen which is well approximated, in a wide range as a straight line (Fig. 7). In ordinary quantum mechanics it is postulated that $P=\Psi^{*} \Psi$, but nothing is said about the kind of detectors that are used for the measurement. In unitary quantum mechanics the statistical interpretation is obtained from the mathematical formalism of the theory. The latter includes the consideration of the problem of the statistical interaction between the particle and the detector and the sensitivity of the latter is accounted for. Since $\rho \approx|\Phi|^{2}$ and in the ordinary formulation of quantum mechanics $P=\Psi^{*} \Psi$ then $|\Phi|^{2}$ and $\Psi^{*} \Psi$ are seen to coincide with an accuracy of terms of the second order. This correction can be verified experimentally as deflections that appear in the contrast of interference and diffraction pictures should be visible. The position of maxima and minima in such pictures cannot, of course, be affected. The most enterprising experimentalists who want to see the light at the end of the tunnel will hopefully check this.

We can easily paraphrase A. Einstein's words about "God playing dice". Now it is quite evident that God does not play each quantum event creating that or another vacuum fluctuation with only one aim: To force the Geiger counter to detect the particle. It is not so absolutely clear that could God do it at all, because for this He should be able tug at all the threads all over the Universe, after careful consideration, and moreover He would need an Ultra-Super-Computer. Apparently God is a perfect mathematician, for He knows Alexander Lyapunov's Central Limit Theorem. That is why He may have decided to make a simple normal distribution of vacuum fluctuations caused by vanishing particles all over the Universe. Two questions remain, however: Was it God who created that Chaos and how did He manage to do it?

\section{The Connection of UQT Equations with Telegraph Equations}

It is known that the current and tension of alternating electric current in pare lines satisfy the telegraph equation that was definitely derived for the first time by Oliver Heaviside from the Maxwell equation. That equation is a relativistic non-invariant which nevertheless lets us see how it corresponds to Quantum Mechanics. The question is that the main relativistic relation between energy, impulse, and mass eq. (3.2) has been still beyond any doubt. Nevertheless, we shall ask ourselves once again about what is happening with that relation at the exact moment when the wave packet disappears being spread over the space. At that moment the particle does not exist as a local formation. This means that in the local sense there is no mass, local impulse, or energy. The particle in that case, within sufficiently small period of time, is essentially non-existent, for it does not interact with anything. Perhaps this is why the relation (3.2) is average and its use at the wavelength level is equal or less than the de Broglie wavelength, which is just illegal. The direct experimental check of that relation at small distances and short intervals is hardly possible today. If the relation (3.2) is declined, then it may result in an additional conservation of energy and impulse refusal; but, as we know, according to the Standard Quantum Theory, that relation may be broken within the limits of uncertainty relation. On the other hand, the Lorenz's transformations have appeared when the transformation properties of Maxwell's equations were analyzing. However electromagnetic waves derived from solutions of Maxwell's equations move all in vacuum with the same velocity, i.e. are not subjected to dispersion and do not possess relativistic invariance. Our partial waves, which form wave packet identified with a particle, possess always the linear dispersion. Under such circumstances, it would be quite freely for author to spread the requirement of relativistic invariance to partial waves. Such requirement has sense in respect only to wave packet's envelope, which appears if we observe a moving wave packet and his disappearance and reappearance. May be the origin of relativistic invariance would be connected in future with the fact that an envelope remains fixed in any reference frames; only the wave's length is changed. 
In the case of periodical vanishing and appearing wave packet (UQT new wave function), taking into account mass oscillation, may be rewritten in the form:

$$
F(x, t)=\exp \left(i \frac{m v^{2}}{\hbar} t\right)[\varphi(x-v t)+\phi(x+v t)],
$$

where packets running in both positive and negative directions $\varphi(x, t)$ and $\phi(x, t)$ are totally arbitrary. For function $F(x, t)$ telegraph equation can be written in the form:

$$
\frac{\partial^{2}}{\partial x^{2}} F(x, t)-\frac{1}{v^{2}} \frac{\partial^{2}}{\partial t^{2}} F(x, t)+2 i \frac{m}{\hbar} \frac{\partial}{\partial t} F(x, t)+\frac{m^{2} v^{2}}{\hbar^{2}} F(x, t)=0
$$

Equations resembling (5.2) may be obtained from Maxwell equations by making a supposition about imaginary resistance of the conductor and using Oliver Heaviside reasoning while deriving from the telegraph equation. However, the equation (5.2) has another solution matching the UQT main idea about the appearing and vanishing packet. That solution (L.Sapogin, 1973, 2005, 2008) has the following form:

$$
F(x, t)=\exp \left( \pm i \frac{m v}{\hbar} x\right) \varphi(x \mp v t)
$$

where we should take the top or bottom sign. Let us write function (5.1) or (5.3) in the form:

$$
F(x, t)=\exp \left(i \frac{m v^{2}}{\hbar} t\right) \Psi(x, t)
$$

or

$$
F(x, t)=\exp \left(i \frac{m v}{\hbar} x\right) \Psi(x, t)
$$

By substituting function (5.5) into the equation (5.2) we get

$$
\exp \left(i \frac{m v^{2}}{\hbar} t\right)\left(v^{2} \frac{\partial^{2}}{\partial x^{2}} \Psi(x, t)-\frac{\partial^{2}}{\partial t^{2}} \Psi(x, t)\right)=0
$$

Reducing the exponential function we get the wave equation. So in the new quantum equation (5.2) O. Heaviside conditions are automatically satisfied (absence of distortion in telegraph equation solution).

Let us insert in our equation (5.2) potential $U(x)$ in a general way. The velocity of the particle with the energy $E$ in a field with potential $U(x)$ may be written as follows:

$$
v=\sqrt{\frac{2(E-U(x))}{m}}
$$

Substituting it into the equation (5.3) and rejecting imaginary terms, we get:

$$
\left[-2 \hbar^{2} E \frac{\partial^{2}}{\partial x^{2}}+2 \hbar^{2} U(x) \frac{\partial^{2}}{\partial x^{2}}+\hbar^{2} m \frac{\partial^{2}}{\partial t^{2}}-4 m E^{2}+8 m E U(x)-4 m U(x)^{2}\right] F(x, t)=0
$$

Let us divide variables in the equation (5.6) in accordance with the standard Fourier technique, assuming that

$$
F(x, t)=\Psi(x) T(t)
$$

After a common substitution in (5.6) and dividing by the product of sought functions we get:

$$
\frac{\hbar^{2}}{\Psi(x)}(U(x)-E) \frac{\partial^{2} \Psi(x)}{\partial x^{2}}+\frac{m \hbar^{2}}{2 T(t)} \frac{\partial^{2} T(t)}{\partial t^{2}}-2 m E^{2}+2 m U(x)(2 E-U(x))=0
$$

After coordinate function $\Psi(x)$ separation and after simple transformations we get the following equation

$$
\frac{U(x)-E}{\Psi(x)}\left[2 m U(x) \Psi(x)-2 m E \Psi(x)-\hbar^{2} \frac{\partial^{2} \Psi(x)}{\partial x^{2}}\right]=0
$$

and we obtain easily the Schrödinger equation: 


$$
\frac{\hbar^{2}}{2 m} \frac{\partial^{2}}{\partial x^{2}} \Psi(x)=(U(x)-E) \Psi(x)
$$

Now substitute function (5.4) into equation (5.2). We obtain

$$
\exp \left(i \frac{m v}{\hbar} x\right)\left[-2 i m v^{3} \frac{\partial \Psi}{\partial x}-\hbar v^{2} \frac{\partial^{2} \Psi}{\partial x^{2}}+\hbar \frac{\partial^{2} \Psi}{\partial t^{2}}-2 i m v^{2} \frac{\partial \Psi}{\partial t}\right]=0 .
$$

By rejecting imaginary terms and reducing we get the wave equation and Heaviside conditions for the absence of distortion are again satisfied. It is curious that while rejecting imaginary terms and requiring $v \rightarrow c$, equation (5.2) is automatically transformed into the Klein-Gordon type equation. All the previously mentioned reasoning can be easily generalized for the three-dimensional case.

It is possible to write down (for the invariance-lover) the following two variants of our telegraph equations:

$$
\frac{1}{v^{2}} \frac{\partial^{2} F(x, t)}{\partial t^{2}}-\frac{\partial^{2} F(x, t)}{\partial x^{2}}+\frac{2 i m c^{2} \sqrt{1-\frac{v^{2}}{c^{2}}}}{\hbar v} \frac{\partial F(x, t)}{\partial x}+\frac{m^{2} c^{4}}{\hbar^{2} v^{2}}\left(1-\frac{v^{2}}{c^{2}}\right) F(x, t)=0
$$

and

$$
\frac{1}{v^{2}} \frac{\partial^{2} F(x, t)}{\partial t^{2}}-\frac{\partial^{2} F(x, t)}{\partial x^{2}}-\frac{2 i m c^{2} \sqrt{1-\frac{v^{2}}{c^{2}}}}{\hbar v^{2}} \frac{\partial F(x, t)}{\partial t}-\frac{m^{2} c^{4}}{\hbar^{2} v^{2}}\left(1-\frac{v^{2}}{c^{2}}\right) F(x, t)=0
$$

These two equations are satisfied exactly by relativistic invariant solutions in the form of a standard planar quantum-mechanical wave and also in the form of disappearing and appearing any wave-packet, viz.

$$
\begin{gathered}
F(x, t)=\exp \left(\frac{i m c^{2} t-m v x}{\hbar} \frac{\sqrt{1-\frac{v^{2}}{c^{2}}}}{\hbar}\right) \\
F(x, t)=\exp \left(\frac{i}{\hbar} \frac{m c^{2} t-m v x}{\sqrt{1-\frac{v^{2}}{c^{2}}}}\right) \varphi(x-v t)
\end{gathered}
$$

The results obtained are quite amazing. It is well known that nearly any equation of theoretically non-quantum physics can result from Maxwell equations. That is why Ludwig Boltzmann said this about Maxwell equations: "It is God who inscribed these signs, didn't He?" Modern science has changed not a semi-point in these equations, and now it appears that even non-relativistic quantum mechanics in the form of the Schrödinger equation may also be extracted from the Maxwell equation. The same can be said about the Klein-Gordon relativistic equation. Moreover, telegraph equations have allowed calculating the spectrum of the masses of the elementary particles (L.Sapogin et al, 2008, 2008, 2010).

\section{The Solution of the Approximate UQT Scalar Equation and the Value of the Fine Structure Constant}

In papers and books (L.Sapogin, 1988, 1991, 2005, 2008), the basic equation (2.8) was reduced to the scalar equation for the density of the space charge of the space charge of the bunch, which represents the particles:

$$
\frac{1}{c} \frac{\partial \Phi(r, t)}{\partial t}+\frac{\partial \Phi(r, t)}{\partial r}+\frac{4 \pi i \Phi(r, t)}{\hbar} \int_{0}^{r}\left\{\Phi^{*}(s, t) \frac{\partial \Phi(s, t)}{\partial t}-\frac{\partial \Phi^{*}(s, t)}{\partial t} \Phi(s, t)\right\} s^{2} d s=0
$$

We seek the solution in the form

$$
\Phi(r, t)=\bar{F}(r) \exp [-\mathrm{i}(\omega t-k r)]
$$

We get the following system of equations if the condition

$$
\omega=k c
$$

is fulfilled: 


$$
\frac{d \bar{F}(r)}{d r}+\frac{8 \pi \omega \bar{F}(r)}{h} \int_{0}^{r} s^{2} \bar{F}^{2}(s) d s=0
$$

Let us suppose

$$
x=\frac{r}{R}, \quad f(x)=\frac{\bar{F}(r)}{\bar{F}(0)}, \quad \bar{F}(0) \neq \infty
$$

Equation (6.3) can be expressed in dimensionless form:

where

$$
\frac{d^{2} \ln f(x)}{d x^{2}}+K x^{2} f^{2}(x)=0
$$

$$
K=\frac{8 \pi \omega R^{4} \bar{F}^{2}(0)}{h}
$$

Solving numerically the Cauchy problem for the eq. (6.4), taking the value $E=16 \pi=2=2=4 \pi$ (where $4 \pi$ from $d V=4 \pi m^{2} d r, 2$ from integral (6.1) and 2 from charge oscillation) and the initial conditions:

$$
f(0)=1, \quad f^{\prime}(0)=0,
$$

we obtain the following integral:

$$
I_{Q}=\int_{0}^{\infty} x^{2} f^{2}(x) d x=8.5137256105758897351 \cdot 10^{-2} \quad I_{Q}{ }^{2}=1 / 137.9623876
$$

The quantity $l_{\mathrm{Q}}$ is a dimensionless electrical charge, which is brought to the following dimensional form:

$$
Q=\sqrt{\hbar c} I_{Q}=4.78709 \cdot 10^{-10} C G S E
$$

This value is less than the modern experimental value of the electron's charge by only $0.3 \%$. This is a fairly accurate number for the first theoretical attempt of the charge calculation. Thus it is not unusual to bring out the "corrections" of the J. Schwinger type to the integral (6.6)

$$
I_{e}=I_{Q}+\frac{I_{Q}^{2}}{8 \pi}-\frac{I_{Q}^{3}}{64 \pi^{2}}=8.54246819177841 \cdot 10^{-2},
$$

which corresponds to the value of charge $e=4.8032514 \cdot 10^{-10}$ CGSE and the value of fine-structure constant $\alpha=1 / 137.0355538109$. The quantization of the electrical charge and masses seems to be the consequence of the balance between the dispersion and nonlinearity, which determines stable solutions.

We regret that we have not succeeded in finding an analytical solution of eq. (6.4), but we are able to give a decent approximation. Let us look for a solution of eq. (6.4) in the form

$$
f(x)=\operatorname{sech} R(x)
$$

Substituting eq. (6.7) into eq. (6.4) and taking into account that for small $R$ we have:

$$
\frac{1}{2} \sinh 2 R \approx R
$$

we obtain

$$
\left(R R^{\prime}\right)^{\prime}=16 \pi x^{2} ; \quad R=\sqrt{\frac{8 \pi}{3}} x^{2} \quad f(x)=\operatorname{sech} \sqrt{\frac{8 \pi}{3}} x^{2}
$$

Author notice that not used any other constants except $\pi$ for calculation of the fine structure constant integration and it had not introduced itself in an underhand way.

\section{Uncertainty relation and principle of complementarity in UQT}

As far as many nonsense have been announced concerning the uncertainty relation we would like to give more detailed of their obtaining first by W.Heisenberg then by N. Bohr and of not quite adequate their interpretation. So, Heisenberg derived the uncertainty relation on well-known now way, now called the method of Heisenberg's 
microscope and based on the analysis of conditions when microparticle's position and motion can be experimentally detected. In principle, the particle's position can be determined by observations of light rays reflected, diffused or emitted by the particle. The particle is considered as a source of light and the results of its observation will be always the diffraction circle with radius equal to the wave length $\lambda$ of this light rays. So the particle position can be determined with precision of order $\lambda$.

The most primitive idea to improve the accuracy of measurements is to use light rays with $\lambda$ being so small as it is possible. It is possible to use, for example, gamma sources, technical implementation of that idea for the time being is not so important. But at the same time we faces A. Compton effect; in the process of measuring the gamma quantum is scattered by the particle and with it the impulse of the particle will be changed for the value equal $h_{h}$ It is paradoxical, but, for example, we will get the same result, for example, in the case of atom while being allocated with the help not of scattered light but of light emitted by atom itself. If the light is emitted in the form of quantum $\hbar \omega$, then atom will receive recoil momentum $\hbar / 2$, and again the study of atoms position will depend on its velocity changes. In both cases the accuracy of atom position determined with the help of scattered or emitted light equals to the wavelength of the light, and momentum change connected with it will be inversely $\lambda$. Increasing the measurements accuracy of particle position, we enlarge the error of definition its momentum. In the result it is impossible to determine the particle momentum at the exact moment of time, when is determined the position of particle since the momentum of particle sharply changes at that very instant. The same considerations would be taken into account at velocity determining also, that resulted in famous Heisenberg relations.

The following philosophical problem appears: is it possible, in principle, to observe any phenomenon without changing it or interfering in it? This problem is no doubt quite old and banal. Anybody agrees that, for example, measuring the electric potential of any object should to change to a certain degree this potential. Any innovations of that measuring apparatus have dealt mainly with tendency to enlarge voltmeter internal resistance and with unachievable idea to make it equal to infinity. Every experimentalist has learned to take into account such non-ideal characteristics of instruments in the process of measuring. And nobody was thrown into confusion with that.

It was proudly announced at the outset of quantum theory that micro-particle does not have at the same moment of time the exact values of co-ordinate and momentum and their values are connected by relation:

$$
\Delta x \cdot \Delta p \geq \hbar
$$

and that statement and that inequality were called as corresponding to nature of micro-worlds objects and quite not caused by lack of appropriate measuring instruments. But the following question may be put: what will happen if within future decades indirect methods possible to use for measuring purposes will be opened? Who is able to foreseen the future?

Shortly after another relation was derived, viz. between energy and moment of time, when that energy being measured:

$$
\Delta E \cdot \Delta t \geq \hbar
$$

That relation appeared in great number of books due to intellectual inertia of some author. And only much later the investigators made out that such relation does not exist within strict quantum mechanics as well as the following relation

$$
t \cdot \hat{H}-\hat{H} \cdot t=i \hbar
$$

does not exist. On the other hand, the operator relation

$$
x \cdot \hat{p_{x}}-\hat{p_{x}} \cdot x=i \hbar
$$

exists and results in uncertainty relation for the coordinate and momentum.

N. Bohr have obtained the same relation after manipulating with wave packets of de Broglie waves (creating a particle from these waves packets), but he had carefully forgotten that these wave packets were spreading. To put it mildly that approach is not quite correct. More over the principle of complementarity offered by Bohr ad hoc, forbade the constructing any speculative models of particle's motion. Since that the main task of the physics became the search of mathematical expressions to be set in one experimental data to obtain the other by 
computations. According to it, the lack of picture in images and motions within quantum physics is not the object of anxiety.

We would rehabilitate the strict standard quantum theory and notice once again that, according to it, the uncertainty relation is obtained as the relation between canonically conjugate additional dynamic variables, and we have nothing to say against. In the essence, the corpuscular - wave dualism became the winner. As we can see now, the uncertainty relation is without any doubts valid but methods used at first for its obtaining were not totally adequate.

UQT overcomes the situation quite easily. As far as the particle (wave packet) is periodically appearing and vanishing at de Broglie wave length (more precisely, the packet disappears twice, and the probability of its detecting is sufficiently big in maximum region only) the position of such a packet may be detected with error

$$
\Delta x \geq \frac{\lambda}{2}
$$

and then

$$
\Delta x \cdot P \geq \frac{h}{2} .
$$

As at measuring of momentum module is inevitable the error $\Delta P=2 P$, then we have following inequality:

$$
\Delta x \cdot \Delta P \geq h
$$

The statements of standard quantum mechanics that particles do not have a trajectory become more understandable. Of course, there is a lot of truth in those words. First, it is possible to say so about intermittent (dotted) motion of the particle with oscillating charge (L.Sapogin 2003, 2005, 2008). Second, any packet (particle) is able during its motion to split into few parts. Each of that parts being summed with vacuum fluctuation may results, in principle, in few new particles. Or visa versa the broken particle may vanish at all and contribute to general fluctuating chaos of the vacuum. But in any case it is better to have more clear idea of particle concrete motion than operate with generally accepted nowadays-obscure sentence about lack of trajectory.

The whole preceding science was based on classical description of objects without taking into consideration material character of the observation process. In other words it was the description of objects or processes "in itself". Quantum science has assigned some limit of such understanding, and although UQT allows describing hypothetically the behavior of quantum objects in "images and motions" there is now either above mentioned hypothetical researchers or their hypothetical experimental devices, and we will have to be content with experimental data obtained with the help of macro-devices.

The principle of complementarity introduced by N.Bohr cannot be explained so easily as it were in the case of uncertainty relation, because it is a set of some philosophical discourses with marks of previous years fight between materialism (it was also called Marxism-Leninism) and other philosophical trends. We would like just now isolate ourselves from any politics, because author do not sympathize politics and philosophical brawls, and tried never to participate in it. Nevertheless, there are objective laws that will not be changed even author and readers disappear, and politicians declare the collapse of materialism and of the said laws. As UQT is able to show many "intimate" sides of quantum behavior and to give the sufficient interpretation of existing quantum processes, the result is quite simple: materialism is gained.

Let us consider rather in more details the principle of complementarity. It is hard to disjoint it from uncertainty relation. Even the origin of its name came from ordinary mechanics, where operators non-commutating with each other correspond to complementary quantities. As we have seen above the uncertainty relation descends from that also. Nevertheless, it is appeared a lot of philosophical explanations which Bohr even had not suspected of. The principle of complementarily can be stated quite popular as follows:

1. A quantum object is extremely complicated formation, not quite easily understood yet, and it's corpuscular and wave characteristics are absolutely unlike and only supplement each other. We can draw rough analogy: maps of Eastern and Western hemispheres, men' photos in full and half face and so on.

2. There are two classes of experimental devices. With the help of ones we can measure the coordinate, the energy and the momentum - the attributes of a particle. With other, while observing the processes of interference or diffraction, one can measure the wavelength. At any measuring (in cases of small energies) particle "is lost" or its parameters change radically in the result of macro situation effect. All that is called as uncontrolled effect that is why it is impossible to measure at the same moment of time corpuscular and wave parameters. 
3. We should not ask Nature questions that will not be experimentally answered.

4. It is not necessary to make attempts in constructing the quantum pictures in images and motions as it were within before-quantum science. It is quite enough to be able mathematically to solve and to analyze different quantum equations and to apply the new rules derived within quantum mechanics. The attitude of Paul Langevin to the last two items was as to something disgusting and he called the principle of complementarity as "intellectual debauchery".

The other numerous statements are based on variants of uncertainty relation.

There were many physical and philosophical discussions about photon behavior at semitransparent mirror (Fig.9). With the help of complementarity principle it was analyzed in what flux (reflected or penetrated) the photon is located while the interference of penetrated or reflected flux is observed and how it correlate with the number of particles to be appeared in penetrated and reflected fluxes. When the flux of particles falling down on the translucent mirror one after another was observed with big exposition, then the interference picture became visible. It contradicts the fact that the particles was detected either in penetrated or reflected flux, and it is incomprehensible how could the interference picture arise. If the particle remains in reflected flux, then it could not been observed in the passed flux, and it is impossible to understand what and with what would interfere. The observed facts of rare simultaneous signals of two particle counters were explained by random appearance of two photons "nearby", and one of them has penetrated the mirror and the other - has reflected. There were some reasons due to observations of induced radiation (that is the main principle the lasers based on). There were made quite enough different experimental variations at that matter. We should note that they are do not contradict the ideas developed within UQT.

Of course not only the processes of splitting cause the phenomena of interference and diffraction. It was shown in (L.Sapogin, 2003, 2005, 2008) that even indivisible particle described by equation with oscillating charge while spreading is able to show the behavior having seemingly a wave character. All these processes look very knotty.

$\mathrm{N}$. Bohr has offered well-known interpretation of that phenomenon from the principle of complementarity viewpoint. We shall remind it shortly. The particles' flow falling down at the mirror is described by wave function (i.e. by the amplitude of probability). The particle after hitting at translucent mirror is, so to say, in a potency state: the particle may belong to penetrate or to reflected flux, it maybe appeared (detected) and maybe not. Namely, that potency is interfering, i.e. possibility of particle's location here or there. These potential possibilities become actual at the finish of object and device interaction only. And though probabilities are referred to potential- possible, i.e. to non-finished experiment, but statistics based on these probabilities is a statistics of realized interactions, i.e. of finished experiments. But if an experimental device would be created being able to follow the destiny of individual particle and to detect to what flux (penetrated or reflected) the particle belong, then the particle would be absorbed or its parameters would be changed at such a value that we would not be able to speak about its participation in interference process. If this process is studied, then it is impossible without violation of interference process to detect the flux, where the photon is located. Either one thing or another, they cannot exist together.

We should note, - it is worthy of astonishment that $N$. Bohr was able to imagine that principle and interpretation, because it turned out that if one follows strictly the prescribed principles and rules, then the right results are obtained and no contradictions arise. All paradoxes were eliminated by simple prohibition to think about it. It stimulated a great philosophical discussion but physicists did not pay attention at. And they were right since that discussion took the form of some talks resulted in nothing, but orthodox quantum interpretation answered every physical question to be asked within new unusual game rules and served as perfect instrument of knowledge. Nevertheless for any thinking researcher the question whether it true raised always. Why we could not even imagine that particle has exact values of momentum and coordinate and follow it dynamics in details? Why we could not study with any indirect methods the concrete sides of particle motion (as it take place in other sciences)?

There are appeared also absolutely new philosophical problems about "free will" and even about the existence of particles in connection with probability interpretation of wave function. Religion was also admixed due to A. Eddington.

There was quite solitary the question about the cause of quantum mechanics statistical character. In connection with that the words of A. Einstein are quoted especially frequently about his unbelief in "God is playing cards". There are so many different speculations about that. But the main is that statistical interpretation does not belong to quantum mechanics instrument and does not result from it but simply postulates. That is not so within 
our UQT and the probability of phenomena appears due to inner content of this theory, and, as we hope, the question about how "God plays cards" has disappeared for most part of our readers at the moment of reading these words.

The author is sure that all additional philosophical quantum-mechanical images of the nature will be crushed down in the nearest future and UQT will gain, and the above mentioned problems will surprise future generation as well as now we are amazed at ancient opinions about three elephants and three whales supporting our Earth. It is astonishing but even these quite naïve ideas had relaxed or rather lulled humanity mind during very long time.

\section{Possible experimental tests and results}

The developed theory will remain a freak of the imagination if the following effects will not be experimentally confirmed:

1. Let very weak source emits by parallel bunch of $N$ particles per $1 \mathrm{sec}$. If the place in front of it is a gate to be opened during the experiment for short interval $\tau \& \frac{1}{N}$, then most probably that no one particle will penetrate or they will be able to do it one by one.

Let these particles fall down on the angle 45 degrees at translucent mirror (Fig.9). According to ordinary quantum mechanics the particle will either penetrate the mirror or reflect. In accordance with the point of view described at that article the bunch will split up at the mirror into two, three... of smaller bunches that depends on bunch phase in front of the mirror and on structure of the mirror in given place. In general we will get two non-similar wave packets (under-thresholds particles or particles converted into state of phantoms) with smaller amplitudes. There are no changes of frequency $\omega$ in formula $E=\hbar \omega$ (reddening), because all processes are linear, i.e. do not depend on amplitude. Besides the particle energy $|\Phi|^{2}$ decreases, that results in reducing the probability of its detecting (considerable vacuum fluctuations is necessary, but the probability of it appearance is too small). So some particles should disappear sometimes during process of measuring or visa versa two particles should appear instead of one. The appearance of two particles from one does not contradict to energy conservation law, as far as the energy of under threshold particle may be increased up to the necessary level due to fluctuations.

Note. A lot of experiments have been carried for example (R.H. Brown, 1957, J. Klauder, 1968) and many others) resulted in conclusions that particles always have distinct tendency to reach detectors in correlated pairs (!) That result confirms we said above. Amusingly, that some physicists have invented special devices of coherent state type for explanation of these experiments refuting standard quantum mechanics. Late the experiments with delayed choice were carried out also confirming the developing in our article point of view. The description of those experiments can be found at "Scientific American" magazine under the title "Quantum philosophy". And quite recently the effect of electron division into two electrons (!) has been experimentally detected (H.Maris, 2000). If those results were true, then it would be the most direct confirmation of UQT and total disaster for the ordinary quantum theory. Unfortunately till now nobody has taken into his head to interpret the results of all such experiments in this way, because energy conservation law formally prohibits it. The last is thoroughly checked at very high levels of energy, and since the energy in that case considerably exceeds the energy of vacuum fluctuation, everything is hold true. But at small energies nobody have studied that question directly. We should repeat once again that any result to be obtained at small energies for one definite particle is random; more over the indeterminateness principle gives no opportunity to detect something precisely for separate particles.

2. The coefficient of passing of any coherent particles with small energies $\left(\lambda_{B} \cong 0.5 A\right)$, through the series of periodical potential barriers (mono-crystal) will be maximal at ( $\lambda_{B}=2 a$ ), where $a$ is the target grid mono-crystal constant (Fig.4). The same, but less weaker effect should appear again at ultra-relativistic energies, when $\Lambda=2 a$. To run such experiments the flux of mono-energetic and synchronous in phase particles is required. It can be obtained by selecting narrow packet of particles reflected from mono-crystal.

3. In connection with the fact that slowly changing part of space-time generates a field, and local hump of that field is a particle periodically disintegrating and appearing, the theory cannot consider processes not satisfying the field laws. Then un-removable vacuum fluctuations really existing will be in such theory non-invariant relative to rotations, transmissions, and space and time reflections and, therefore, conservation laws concerned with them will be non-local and approximate. Such infringements easily arise when particle energy $\mid \boldsymbol{\rho}^{2}$ is of the same range as dispersion $\sigma$ of vacuum fluctuations is. Unfortunately, these processes will arise near the threshold and therefore they are difficult for investigation. 
4. Since every particle can spontaneously arise from vacuum or vanish with very small probability, all chemical elements are subjected to absolutely new type of nuclear transformations: any element may be transformed into his isotope or into one of his nearest neighbour in periodic table. Upon a time, (E.Rutherford, 1905) pointed it out, and these processes were really discovered in geology, but they have no explanations.

5. At collision of any particles the processes of mutual penetration without any other interaction are to be detected in the case when in the point of collision one of particles or both will spread. It seems, $s-$ state of hydrogen atom is a good illustration of that. We should note that the same phenomena have appeared in Bohr-Sommerfeld model (pendulum orbits) too, but were rejected at once by standard quantum theory as quite preposterous.

6. The any particle is a moving wave packet. From mathematical perspective after Fourier transformation our packet equivalents endless set of flat harmonic waves, which nowhere begin and nowhere end. If a medium with strong dispersion is placed on the way of these waves (L.Wang, 2000, see Fig.10), then it can result a particle can appear, though, nothing do not moving (!). In UQT also no any limits for velocities of the particles! But on the other hand, usual using the determination to velocities in the UQT not applicable. Let's to entrust the mathematician! During the last several years many groups have experimentally confirmed possibility of superluminal light propagation. This should be considered as direct experimental proof of principle of UQT.

7. New wave from eq. (3.1) for Stanford-SLAC $\Lambda=120 \AA$ and easy can find.

\section{Conclusion}

It would be appropriate to mention one more statement of one of quantum theory founders (quite disavowing this theory, but almost unknown - why? - among broad scientific community):

"There are many experiments that we are just not able to explain if we don't consider the waves as namely waves exerting its influence upon all region, where they spread, and assume the location of these waves being "possibly here, possibly there according to probabilistic viewpoint". E. Schrödinger, Brit.J.Philos.Sci, vol.3, page 233, section 11, 1952.

In conclusion it would be relevant to mention that Louis de Broglie predicted this discovery: "Those who say that new interpretation is not necessary I would like to note that new interpretation may have more deep roots and such theory in the long run will be able to explain wave-particle dualism, but that explanation will not be received either from abstract formalism, modern nowadays, or from vague notion of supplementary. But I think that the highest aim of the science is always to understand. The history of the science shows if any time somebody succeeded in deeper understanding of physical phenomena class, new phenomena and applications appeared. Hope that many researchers will study that enthralling question casting aside preconceived opinions and not overestimating the importance of mathematical formalism, whatever beautiful and essential it was, because that may result in loss of deep physical sense of phenomena” Louis de Broglie, Compt. Rend, 258, 6345, 1964.

The offered outline of unitary quantum mechanics for a single particle from the position of united field is rather simple and obvious from hypothetical observer's point of view. If a hypothetical observer usually can measure the value of the wave function amplitude, we cannot do it at all. We have to be satisfied with its probability interpretation keeping in mind that rather very simple mechanism is hidden behind and this mechanism open the way for explanation of quality transformations of quantum phenomena, and allows to reduce the description of the whole nature to description of some united field, and the continuous transformations of that field show the astonishing variety of phenomena being under observation.

In spite of mathematical complexity Unitary Quantum Theory will stop being paradoxical and frank words of Richard Feynman "I can easily say that nobody understands quantum mechanics" will become the property of history.

\section{Acknowledgements}

The author are thankful to cosmonaut, R.F. Air Force General Vladimir A. Dzhanibekov, to Professors V.A.Boichenko, A.S. Bogomolov (Moscow), V.M. Dubovik (Dubna, JINR), P.I. Pospelov (Moscow), V.M. Prihod'ko (Moscow), Yu.A.Ryabov, and translator S.V. Romanova for support of our work and fruitful discussions. 


\section{References}

Alexandrov A.D., Fock V.A. (1956). Philosophical Questions of Modern Physics, in Russian, Kiev.

Boichenko V.A., Sapogin L.G. (1984). On the Equation of the Unitary Quantum Theory. Annales de la Fondation Louis de Broglie, vol. 9, No.3, p.221.

Brown H. etc. (1957). Proc.Roy.Soc, A243, 291.

Darwin C.G. (1927). Proc.Roy.Soc., A117, p.258.

Feynman R. etc. (1965). Quantum Mechanics and Path Integrals, McGraw-Hill, New York.

Heisenberg W. (1966). Introduction to the Unified Field Theory of Elementary Particles, Interscience, London, New York, Sydney.

Klauder J.R. etc. (1968). Fundamentals of Quantum Optics”,W.A.Benjamin, Inc.,New York, Amsterdam.

Liu W., et al. (1999). Phys. Rev. Lett. v. 82, 711.

Maris H.J., (2000). On the Fission of Elementary Particles and the Evidence for the Fractional Electrons in Liquid Helium. Journal of Low Temperature Physics, vol.120, page 173.

O.Costa de Beauregard. (1957). Theorie Synthetique de la Relativite Restreinte et des Quanta (Gauther-Villars, Paris, 1957).

Rutherford E. (1905). Pop. Sci. Monthly, N.Y.77, 5.

Sapogin L.G. (1973). United field and Quantum Mechanics, System Researches (physical Researches) Acad. Science USSR, Vladivostok, 2, pp. 54-84, (Russian).

Sapogin L.G. (1979). On Unitary Quantum Mechanics. Nuovo Cimento, vol. 53A, No 2, p.251.

Sapogin L.G. (1980). A Unitary Quantum Field Theory. Annales de la Fondation Louis de Broglie, vol.5, No 4, p.285-300.

Sapogin L.G. (1982). A Statistical Theory of Measurements in Unitary Quantum Mechanics. Nuovo Cimento, vol.70B, No.1, p.80.

Sapogin L.G. (1982). A Statistical Theory of the Detector in Unitary Quantum Mechanics. Nuovo Cimento, vol.71B, No. 3, p.246.

Sapogin L.G., Boichenko V.A. (1988). On the Solution of One Non-linear Equation. Nuovo Cimento, vol.102B, No 4, p.433.

Sapogin L.G., Boichenko V.A. (1991). On the Charge and Mass of Particles in Unitary Quantum Theory. Nuovo Cimento, vol.104A, No 10, p.1483.

Sapogin L.G., Ryabov Yu. A. (2008). On the mass spectrum of elementary particles in Unitary Quantum Theory, The Old and New Concepts of Physics, Vol. 5, No 3.

Sapogin L.G., Ryabov Yu. A. (2010). New Theoretical Results about the Mass Spectrum of Elementary Particles. Applied Physics Research, vol. 2, No 1, p.86-98, May.

Sapogin L.G., Ryabov Yu. A., Boichenko V. A. (2008). Unitary Quantum Theory and a New Sources of Energy, Ed. Science-Press, Moscow, (Russian, transl. from English).

Sapogin L.G., Ryabov Yu.A, Boichenko V.A. (2005). Unitary Quantum Theory and a New Sources of Energy, Archer Enterprises, Geneva, NY, USA.

Sapogin L.G., Ryabov Yu.A., Utchastkin V.I. (2003). Unitary Quantum Theory and a New Energy Sources. Ed. MADI, Moscow, (Russian).

Wang L.J. etc. (2000). Gain-assisted superluminal light propagation, Nature, 406, p.277-279. 


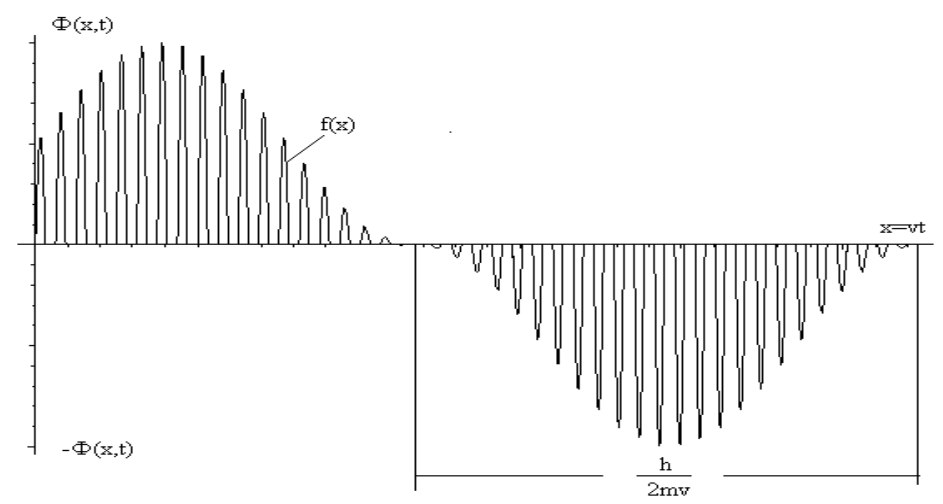

Figure 1. Behaviour of wave packet in linear dispersion medium (i.e., rather like a series of stroboscopic photographs).

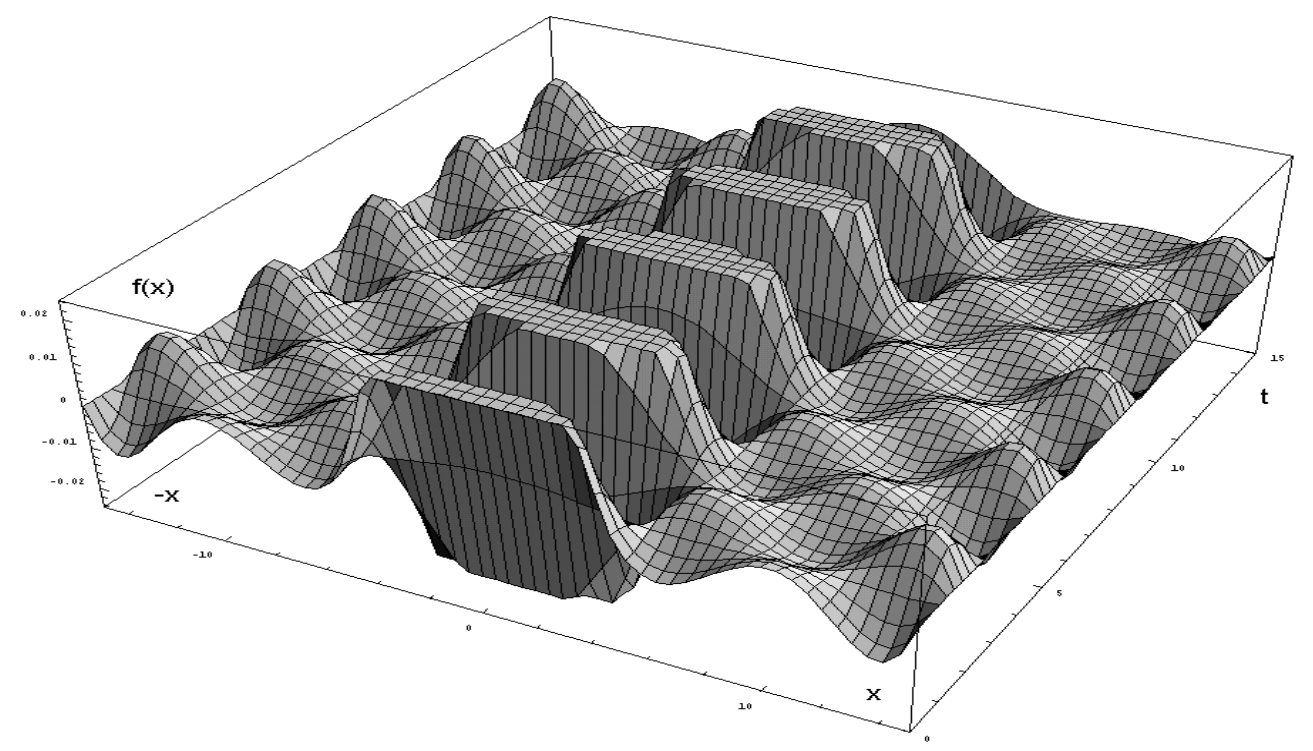

Figure 2. Mathematical modeling of Gauss packet behaviour

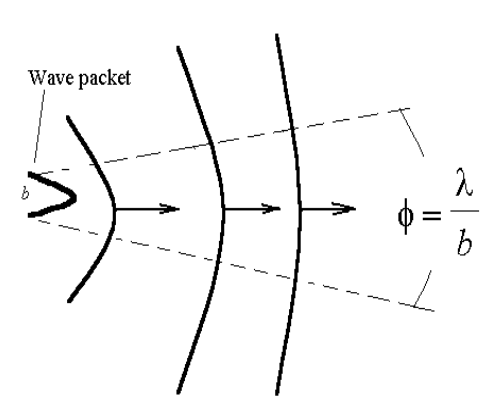

a

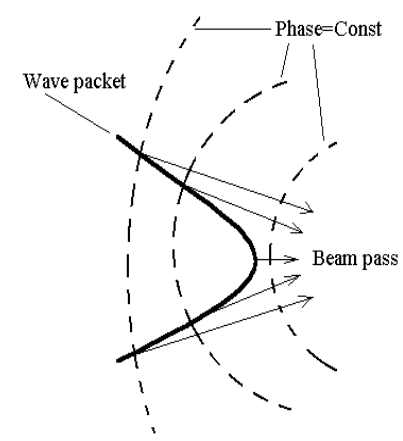

b

Figure 3. Wave packet dispersion and refocusing 


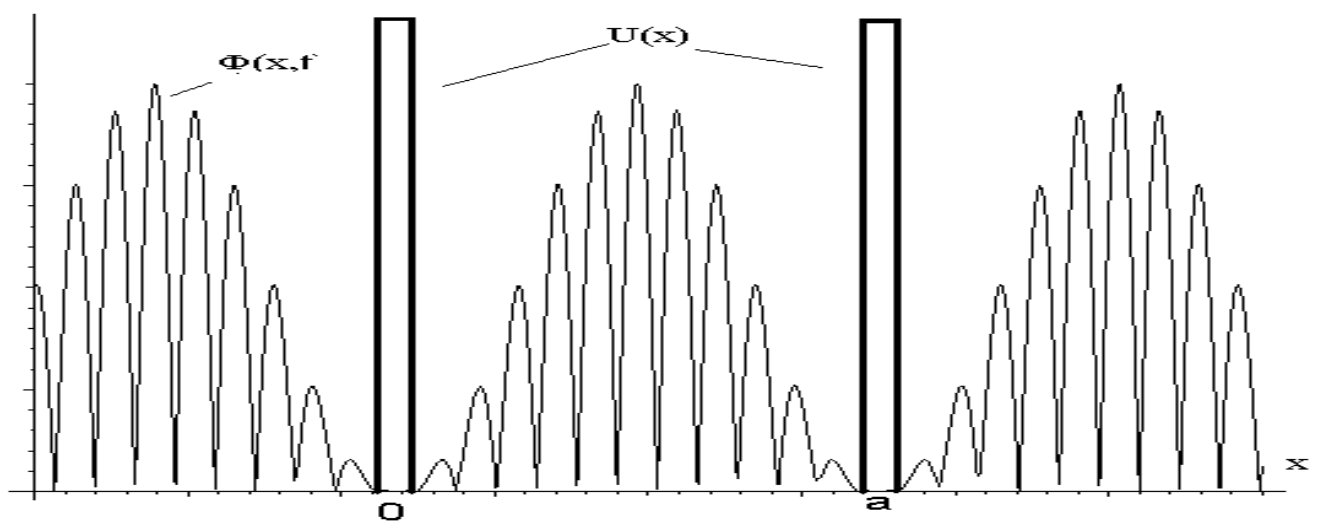

Figure 4.
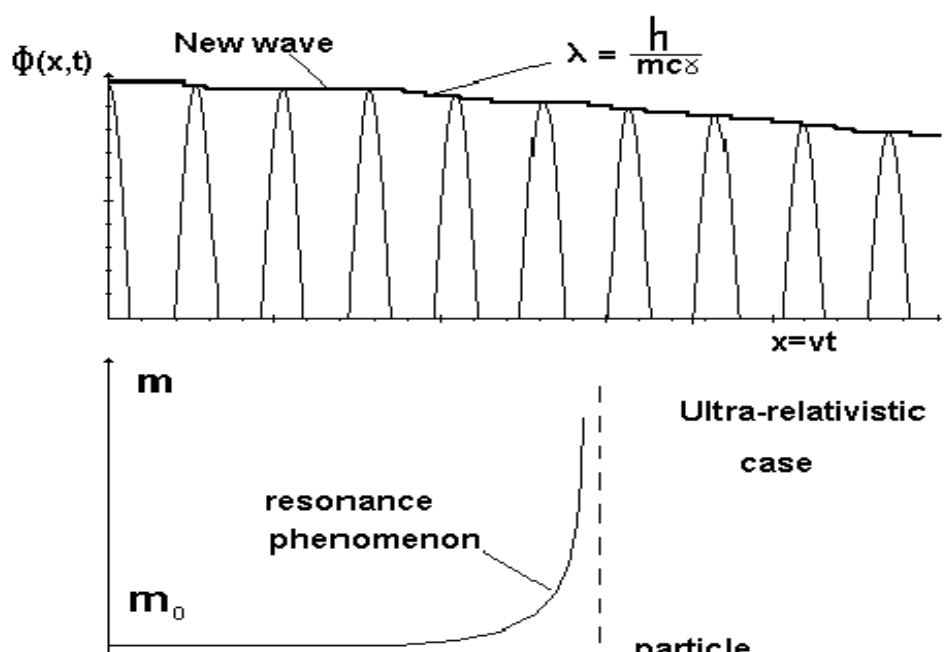
Ultra-relativistic

case

Within the ultra-relativistic limit the wave length $\lambda$ be comes much greater than the characteristic dimension of the quantum system with wich it interacts. Therefore, the particle stationary wave packet moving in accordance with the classical laws.

Figure 5

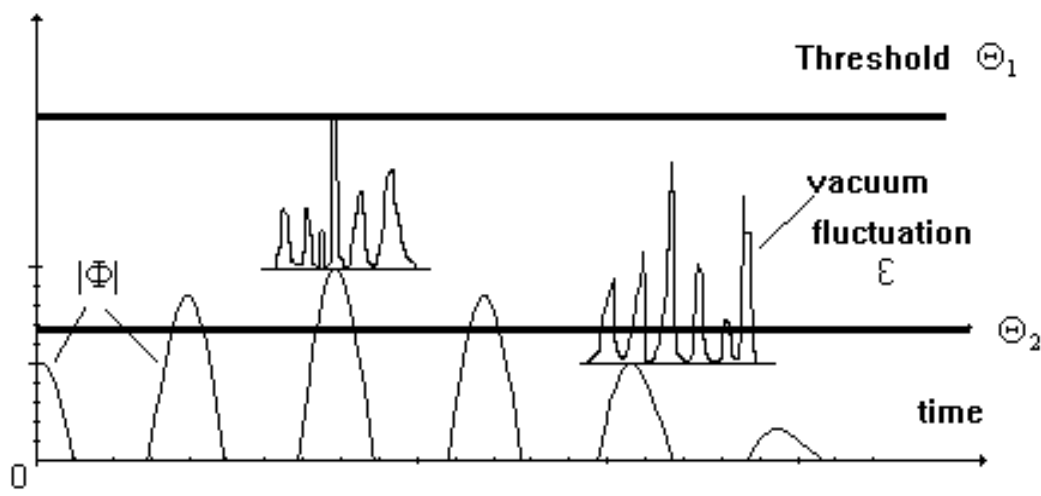

Figure 6. 


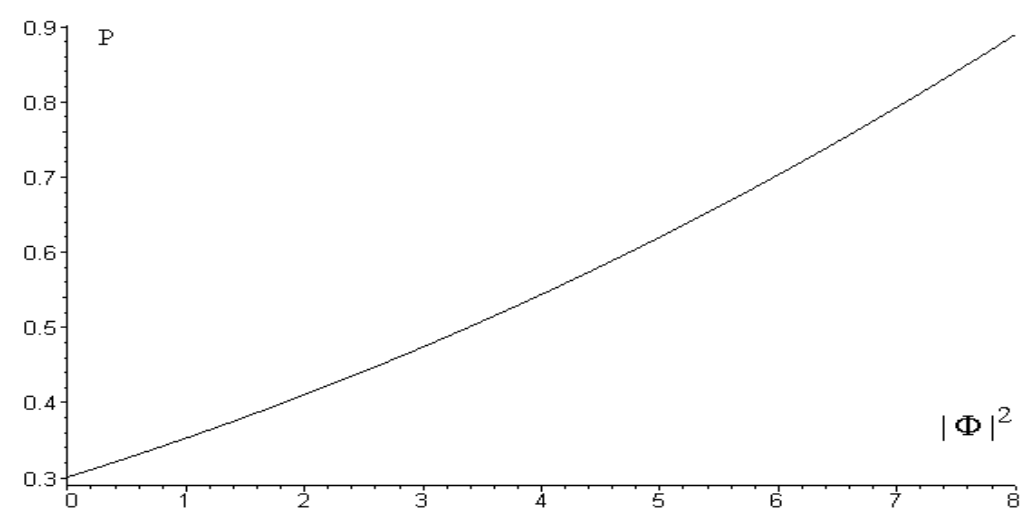

Figure 7. Probability of regular detection of particle as a function of $|\Phi|^{2}$

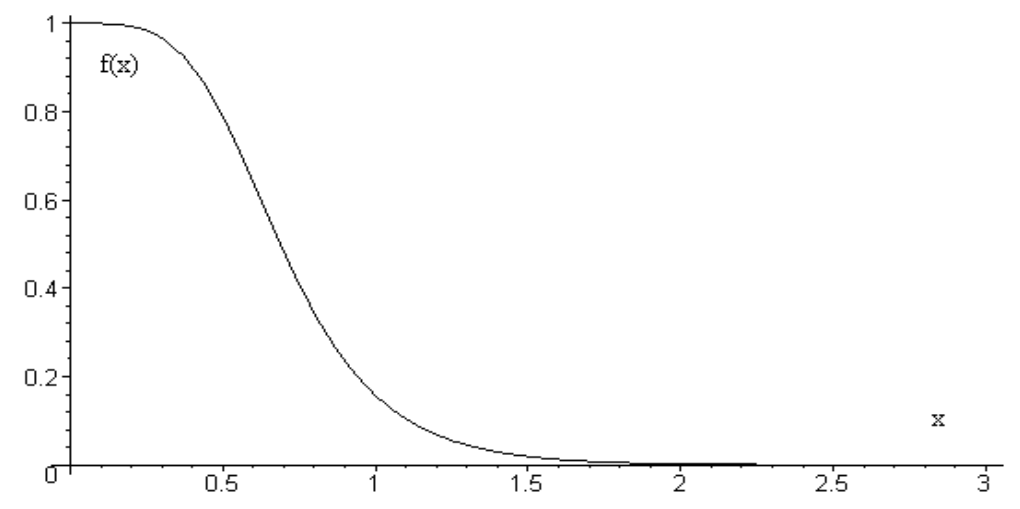

Figure 8. Shows diagrams for the equation computational solution eq.(6.4).

Particles

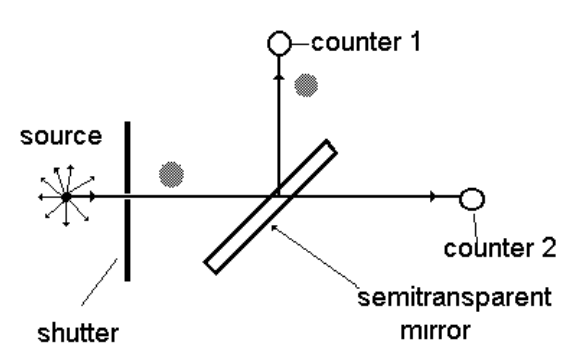

Waves

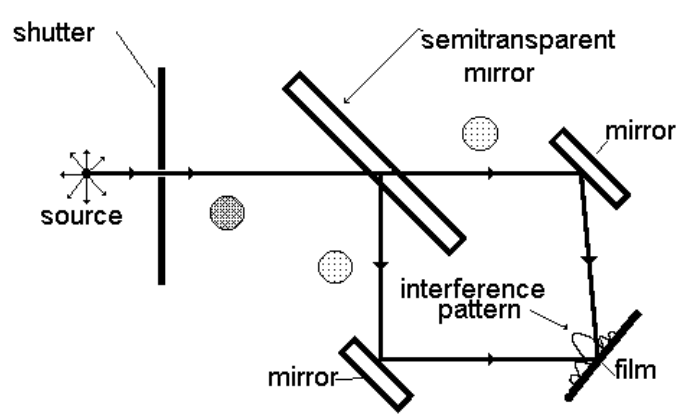

Figure 9. Experiments with individual photons on semitransparent mirror

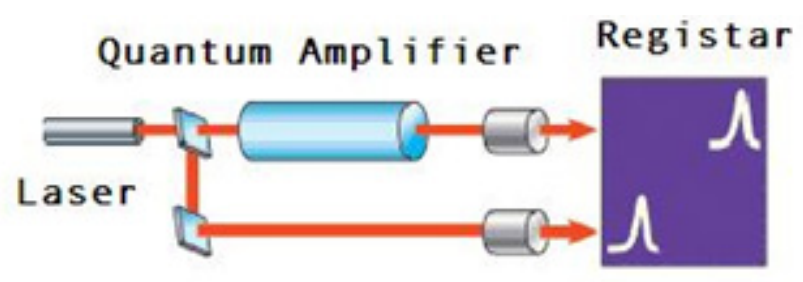

Photoreceiver

Figure 10. Experiments of L.Wang - superluminal light propagation 